\title{
Rate-One Space-Frequency Block Codes With Maximum Diversity for MIMO-OFDM
}

\author{
Lei Shao, Member, IEEE, and Sumit Roy, Senior Member, IEEE
}

\begin{abstract}
We propose a novel rate-one (i.e., one symbol per transmission), space-frequency block code (SFBC) for an orthogonal frequency division multiplexing (OFDM) system with transmit $(M)$, and receive $(N)$ antennas that achieves the maximum diversity attainable over frequency-selective channels. Moreover, the space-frequency (SF) code design is shown to be robust to overestimation of the (true) channel order $L$ at the price of increasing decoding complexity. Further, even if the channel order is underestimated $(K<L)$, diversity order of $M N K$ is achieved with commensurately reduced decoding complexity; this allows a natural mechanism for trading diversity for decoding complexity as desired. In addition, since the SF code symbol is transmitted in one OFDM block duration, it has a smaller processing delay than comparable space-time-frequency block codes (STFBC).
\end{abstract}

Index Terms-MIMO, OFDM, space-frequency block codes, space-time block codes.

\section{INTRODUCTION}

$\mathbf{F}$ IXED broadband wireless access (BWA) in the $2-11 \mathrm{GHz}$ band is a rapidly maturing "last-mile" solution for residential and enterprise scenarios that holds great promise for regions with low penetration of cable/telephony loop plant. The IEEE 802.16a Task Group for Wireless Metropolitan Area Networks has recently finalized a standard [1], [2] for wireless access to an external stationary receiver on customer premises from a nearby base station.

In fixed BWA, deep fades due to multipath propagation can persist over a significant period of time and such wide-area wireless channels also encounter significant multipath dispersion that limits the maximum achievable rates. Since fixed BWA competes with cable modems and asynchronous digital subscriber line where the channel is static nonfading, new system designs must counteract these key challenges and provide high data-rate access comparable to wireline cost/performance considerations.

The use of multiple antennas at both transmit and receive ends of a wireless link resulting in a multiple input multiple output (MIMO) channel has been recognized as an important system component towards meeting these requirements. Pointto-point MIMO schemes ideally allow both high-rate trans-

Manuscript received May 13, 2003; revised November 4, 2003; accepted May 10, 2004. The editor coordinating the review of this paper and approving it for publication is $\mathrm{H}$. Boelcskei. This research was supported by NSF/ITR under CCR 0086032.

L. Shao was with University of Washington. She is now with Intel Corporation in Santa Clara, CA (e-mail: lei.shao@intel.com).

S. Roy is with the Department of Electrical Engineering, University of Washington, Seattle, WA 98195 USA (e-mail: roy@ee.washington.edu).

Digital Object Identifier 10.1109/TWC.2005.850374 mission and exploitation of the multipath diversity available between any transmit-receive pair in an MIMO setup; in practice there exists a fundamental tradeoff between spatial multiplexing that increases the achievable rate and diversity that enhances link reliability [9].

As is well known, orthogonal frequency division multiplexing (OFDM) is a multicarrier transmission scheme that can potentially attain high-rate transmission due to enhanced temporal spectral efficiency. In packetized transmission over frequencyselective (dispersive) channels, OFDM can eliminate the effects of ISI by inserting cyclic prefix (CP) in the header, at the cost of a penalty in channel utilization due to the added overhead. Hence, receive equalization to compensate for the channel frequency response (probably the most important receiver subsystem in single-carrier modulation) is now trivially implemented via a complex scalar multiplication on a tone-by-tone basis. Thus a combination of MIMO and OFDM (MIMO-OFDM) appears particularly promising for fixed BWA. In this paper, we focus on space-time (S-T) coding methods that maximize diversity gain in MIMO-OFDM systems over frequencyselective channels, to meet the quality of service requirements and desired data rates for intended applications.

\section{A. Literature Review}

S-T coding was initially introduced for narrowband singlecarrier systems operating over flat fading channels where spatial diversity in the form of multiple transmit antennas was available. The simple linear block code of [15] was generalized via the theory of S-T block codes (STBCs) [18], but at the price of rate loss for a complex constellation and when the number of transmit antennas exceed two. To maintain rate of unity, nonorthogonal STBC has been proposed by several authors (e.g., [27], [29], and [30]) which trades decoding complexity for rate-one. Moreover, $\mathrm{S}-\mathrm{T}$ block coding based on linear complex field (LCF) precoding enables full diversity without any rate loss [7], [8], [22], [26], [28]. For broadband wireless systems over frequency-selective fading channels, S-T code (STC)OFDM modulation [10], [13] ${ }^{1}$ has been naturally proposed and STBC-OFDM can preserve the orthogonality of STBCs (that leads to a simple receiver) while enabling high data rates. However, the channels need to be quasistatic over several OFDM symbol blocks. Moreover, since the S-T encoding is applied independently on subcarriers, only maximum spatial diversity

\footnotetext{
${ }^{1}$ Here, each subcarrier is independently S-T encoded and the resulting symbols sent over multiple transmit antennas.
} 
but not frequency diversity is achieved. In order to exploit the inherent frequency selectivity available in the channel, coding across the subcarriers is needed. Several S-T-frequency (STF) or space-frequency (SF) schemes have been proposed [3]-[6], [12], [14], [20]. However, simply using Alamouti code on adjacent subcarriers [12] fails to exploit the frequency selectivity in the channel. Similarly, [14] trades achievable rate for limited (second order) frequency diversity. In addition, in [14], the choice of the cyclic shift of the replica symbol depends on the channel, necessitating feedback.

In [6], the S-T trellis code designed shows that a large effective block length and the ideal interleaving are two key requirements in STC coding for OFDM systems. The authors adopt existing codes that do not achieve the maximum diversity gain available in the channel.

In [3], the design criteria for SF coding was established. It was shown that the figure of merit for designing good SF codes is vastly different from that for $\mathrm{S}-\mathrm{T}$ codes in narrowband fading channels. For example, employing known $\mathrm{S}-\mathrm{T}$ codes as SF codes by coding across space and frequency (rather than across space and time) in general provides spatial diversity but fails to exploit the available frequency diversity [3]. The case of two transmit and one receive antennas for a two-tap channel was considered in [4]. Although a full diversity code was designed, the design criteria imposed limits on the achievable code rate leading to a rate of 1/4 SF code. Most recently, [32] proposed a systematic method for the design of SF codes with variable multiplexing-diversity tradeoff through linear precoding.

In [5], a novel STF block code for a multiple-antenna OFDM transmission over frequency-selective Rayleigh fading channels was proposed. Incorporating subcarrier grouping and choosing appropriate system parameters, the authors converted their system into a set of grouped STF systems. This enabled simplified STF block coding within each group. The resulting codes were shown capable of achieving both maximum diversity and coding gain, while affording low-complexity decoding. However, since the authors used orthogonal STBC as the component S-T code, STFBC incurs rate loss when the number of transmit antennas is greater than 2. Another disadvantage of [5] is that it requires the channel to be constant during $2 M(M \geq 4)$ OFDM symbol times for $M$ transmit antennas implying a longer processing delay. Recently, another kind of S-T-multipath coding method, which uses digital phase sweeping (DPS), has been proposed in [20]. This overcomes the drawback of rate loss in [5], guarantees maximum diversity, and achieves good coding gain.

The contribution of this paper is a new SF block coding scheme that achieves maximum diversity available in MIMOOFDM systems over frequency-selective channels; like [20], this maximum space-multipath diversity is obtained without rate loss for any number of transmit antennas and requires that the channel be constant only for one OFDM symbol duration. We note that while [20] and our SFBC-OFDM were designed independently resulting in very different encoder structures, the complexity and performance of these two schemes are very close to each other. Table I summarizes the above discussion and provides a comparison among various ST/SF/STF blockcoded OFDM schemes.
TABLE I

COMPARISON AMONG VARIOUS ST/SF/STF BLOCK CODED OFDM SCHEMES

\begin{tabular}{c||c||c||c}
\hline Methods & $\begin{array}{c}\text { Diversity } \\
\text { (DG) }\end{array}$ & $\begin{array}{c}\text { Code Rate } \\
\text { (CR) }\end{array}$ & Comments \\
\hline \hline STBC-OFDM & $2 \mathrm{~N}$ & 1 & if $M>2$, \\
$(\mathrm{M}=2)$ & & & $D G=M N$ \\
SFBC-OFDM[12] & $2 \mathrm{~N}$ & 1 & if $M>2$, \\
$(\mathrm{M}=2)$ & & & $D G=M N$ \\
& & & $C R<3 / 4$ \\
STFBC-OFDM[14] & $4 \mathrm{~N}$ & 0.5 & if $M>2$, \\
$(\mathrm{M}=2)$ & & & $D G=2 M N$ \\
& & & $C R<0.5$ \\
STFBC-OFDM[5] & $2 \mathrm{NL}$ & 1 & if $M>2$, \\
$(\mathrm{M}=2)$ & & & $D G=M N L$ \\
& & & $C R<3 / 4$ \\
DPS[20] & MNL & 1 & $\forall M, N$ \\
SFBC-OFDM proposed & MNL & 1 & $\forall M, N$ \\
\hline
\end{tabular}

\section{Design Criteria And Code Construction}

We adopt the design criteria proposed in [3] for an MIMO-OFDM system with $M$ transmit and $N$ receive antennas and $N_{\mathrm{c}}$ subcarriers, where $N_{\mathrm{c}} \gg M, N$. Let $\mathbf{C}=$ $\left[\begin{array}{llll}\mathbf{c}_{0} & \mathbf{c}_{1} & \ldots & \mathbf{c}_{N_{\mathrm{c}}-1}\end{array}\right]$ and $\mathbf{E}=\left[\begin{array}{llll}\mathbf{e}_{0} & \mathbf{e}_{1} & \ldots & \mathbf{e}_{N_{\mathrm{c}}-1}\end{array}\right]$ be two different SF code words represented by matrices of size $M \times N_{\mathrm{c}}$, where $\mathbf{c}_{k}=\left[\begin{array}{llll}c_{k}^{(0)} & c_{k}^{(1)} & \ldots & c_{k}^{(M-1)}\end{array}\right]^{\mathrm{T}}$ stands for the $k$ th frequency vector composed of transmitted data symbols with $c_{k}^{(i)}$ denoting the data symbol transmitted from the $i$ th antenna on the $k$ th tone. In [3], it is assumed that the channel consists of $L$ matrix taps (each of size $N \times M$ ) with the matrixvalued transfer function given by

$$
\mathbf{H}\left(e^{j 2 \pi \theta}\right)=\sum_{l=0}^{L-1} \mathbf{H}_{l} e^{-j 2 \pi l \theta}, \quad 0 \leq \theta<1 .
$$

For Rayleigh fading channels, the elements of $\mathbf{H}_{l}(l=$ $0,1, \ldots, L-1)$ are (possibly correlated) circularly symmetric zero-mean complex Gaussian random variables with variance $\sigma_{l}^{2}$, where the path gain $\sigma_{l}^{2}$ is derived from the power delay profile of the channel. Furthermore, it is assumed that heavy scattering occurs around the transmitter whereas the receiver is unobstructed. This implies that the signal from different transmit antennas will be uncorrelated, modeled by assuming that different columns of $\mathbf{H}_{l}$ are uncorrelated. Due to the lack of scattering at the receiver, signals at different receive antennas will be correlated which corresponds to correlation among different rows of $\mathbf{H}_{l}$. In addition, the contributions from distinct scattering clusters are assumed to be uncorrelated and a uniform linear array is used for both the transmitter and the receiver. 
An upper bound on the expected pairwise error probability (averaged over all channel realizations) was derived in [3] as

$$
P(C \rightarrow E) \leq \prod_{i=0}^{\operatorname{rank}\left(C_{Y}\right)-1}\left[1+\lambda_{i}\left(C_{Y}\right) \frac{E_{s}}{4 \sigma_{n}^{2}}\right]^{-1}
$$

where $\quad C_{Y}=\sum_{l=0}^{L-1}\left[D^{l}(C-E)^{\mathrm{T}}(C-E)^{*} D^{l^{\mathrm{H}}}\right] \otimes \mathbf{R}_{l}=$ $F(C, E) F(C, E)^{\mathrm{H}}$ has a dimension $N_{\mathrm{c}} N \times N_{\mathrm{c}} N$ and $\lambda_{i}\left(C_{Y}\right)$ is the $i$ th nonzero eigenvalue of $C_{Y} . \mathbf{R}_{l}$ denotes the $N \times N$ correlation matrix of the $k$ th column of the zero-mean matrix $\mathbf{H}_{l}$, which is independent of $k$, or equivalently the fading statistics are the same for all transmit antennas. Also, the power delay profile of the channel is absorbed into the correlation matrices. $F(C, E)$ is an $N N_{\mathrm{c}} \times M N L$ matrix defined as shown in (3) at the bottom of the page, where $D=$ $\operatorname{diag}\left\{\exp \left[-j\left(2 \pi / N_{\mathrm{c}}\right) k\right]\right\}_{k=0}^{N_{\mathrm{c}}-1}$. As mentioned in [3], the achievable diversity order critically depends on the propagation environment and the antenna spacing (interested readers should refer to [25] for details on the impact of receive correlation on $\mathrm{SF}$ coded systems). In order to achieve $M N L$-fold diversity, it is necessary that: 1) $r\left(\mathbf{R}_{l}\right)=N$ for $l=0,1, \ldots, L-1$;2) the $N_{\mathrm{c}} \times M$ matrix $(C-E)^{\mathrm{T}}$ is full rank over all distinct $\{C, E\}$ pairs; and 3) the stacked matrix $F(C, E)$ always has full rank as well.

It is already known that for the case when $\mathbf{R}_{l}$ is full rank, the diversity order achieved by an SF code is independent of spatial correlation (i.e., the specific structure of $\mathbf{R}_{l}$ ); ${ }^{2}$ hence without loss of generality, we assume in the subsequent proof that the channel is characterized by: i) no spatial fading correlation and ii) uniform power delay profile, i.e., $\mathbf{R}_{l}=I_{N}$ for $l=$ $0,1, \ldots, L-1$.

For the above, $C_{Y}$ can be simplified as $C_{Y}=S \otimes$ $I_{N}$ with $S=G(C, E) G^{\mathrm{H}}(C, E)$ having a dimension $N_{\mathrm{c}} \times$ $N_{\mathrm{c}}$ where $G(C, E)$ is the $N_{\mathrm{c}} \times M L$ matrix $G(C, E)=$ $\left[\begin{array}{llll}(C-E)^{\mathrm{T}} & D(C-E)^{\mathrm{T}} & \cdots & D^{L-1}(C-E)^{\mathrm{T}}\end{array}\right]$. Therefore, the upper bound in (2) on the expected pairwise error probability (averaged over all channel realizations) simplifies to [4]

$$
P(C \rightarrow E) \leq \prod_{i=0}^{\operatorname{rank}(S)-1}\left[1+\lambda_{i}(S) \frac{\rho}{4}\right]^{-N}
$$

${ }^{2}$ Note that receiver spatial correlation does induce a loss of coding gain, but this is independent of the code design [25]. where $\rho=\left(E_{s} / \sigma_{n}^{2}\right)$ is the average signal-to-noise ratio (SNR) and $\lambda_{i}(S)$ is the $i$ th nonzero eigenvalue of $S$. The diversity order of the code is defined as $G_{d}=\min _{\{C, E\}} \operatorname{rank}(S) \times N$. For $N_{\mathrm{c}}>M L$, the rank of the $N_{\mathrm{c}} \times M L$ matrix $G(C, E)$ is at most $M L$ and hence the maximum achievable diversity order is $M N L$. In order to achieve $M N L$-fold diversity, appropriate code design is needed to ensure that not only the $M \times N_{\mathrm{c}}$ error matrix $(C-E)$ is full rank over all distinct $\{C, E\}$ pairs, but the stacked matrix $G(C, E)$ always has full rank as well.

\section{A. Code Construction}

For rate-one SF block codes, the number of information symbols mapped into the $\mathrm{SF}$ code matrix is equal to the number of subcarriers $N_{\mathrm{c}}$. We choose $N_{\mathrm{c}}=M \times K \times G$, where $K$ is a transmitter design parameter that can be appropriately adjusted. To achieve full diversity order $M N L$, we can choose $K \geq L$; however, we may choose to use $K<L$ to trade diversity with lower receiver complexity. In addition, $G$ is the number of groups the subcarriers are divided into (see Figs. 1 and 2).

First, the $N_{\mathrm{c}} \times 1$ vector of input symbols $\mathbf{s}=$ $\left[\begin{array}{llll}\mathbf{s}_{0}^{\mathrm{T}} & \mathbf{s}_{1}^{\mathrm{T}} & \cdots & \mathbf{s}_{G-1}^{\mathrm{T}}\end{array}\right]^{\mathrm{T}}$ is divided into $G$ groups of size $M K \times 1$ vectors $\left\{\mathbf{s}_{g}\right\}_{g=0}^{G-1}$. Next, each $\mathbf{s}_{g}$ is left multiplied by the same constellation rotation (CR) precoder $\Theta$ [8] of dimension $M K \times M K$ to produce size $M K$-vector $\mathbf{v}_{g}=\Theta \mathbf{s}_{g}=$ $\left[\begin{array}{lll}\boldsymbol{\theta}_{1}^{\mathrm{T}} \mathbf{s}_{g} & \cdots & \boldsymbol{\theta}_{M K}^{\mathrm{T}} \mathbf{s}_{g}\end{array}\right]^{\mathrm{T}}$, where $\boldsymbol{\theta}_{i}^{\mathrm{T}}$ denotes the $i$ th row of $\Theta$. Then we partition each $\mathbf{v}_{g}$ into $K \quad M \times 1$ subvectors; each subvector is then used to create an $M \times M$ diagonal ma$\operatorname{trix} D_{\mathbf{s}_{g}, k}=\operatorname{diag}\left\{\boldsymbol{\theta}_{M \times(k-1)+1}^{\mathrm{T}} \mathbf{s}_{g} \quad \cdots \quad \boldsymbol{\theta}_{M \times k}^{\mathrm{T}} \mathbf{s}_{g}\right\}$, for $k=$ $1, \ldots, K$. The resulting $K$ submatrices are regarded as belonging to the same group. Finally, we map the submatrices from the $G$ groups (a total of $G \times K$ diagonal matrices) into a large $M \times N_{\mathrm{c}} \mathrm{SF}$ matrix in such a way so that submatrices from the same group are equally spaced. In other words, a total of $G$ precoded symbol vectors $\left\{\mathbf{v}_{g}\right\}_{g=0}^{G-1}$ are mapped in the SF domain to get the $M \times N_{\mathrm{c}} \mathrm{SF}$ matrix (or SF codeword) [see (4a) at the bottom of the page]

Remarks:

1) Generally, the true number of channel taps $L$ is unknown at the transmitter, which assumes a parameter $K \neq L$ for this purpose. Implicitly, we assume that $K$ is also known at the receiver.

2) Introduction of $G$ is needed to ensure $N_{\mathrm{c}}=M K G$ holds since generally $M L<N_{\mathrm{c}}$ and the design parameter $K$ related to $L$ is generally chosen to satisfy $M K<$ $N_{\mathrm{c}}$. Typically, $G$ will be small $(G=1$ when $M K$ is

$$
F(C, E)=\left[\begin{array}{llll}
(C-E)^{\mathrm{T}} \otimes \mathbf{R}_{0}^{\frac{1}{2}} & {\left[D(C-E)^{\mathrm{T}}\right] \otimes \mathbf{R}_{1}^{\frac{1}{2}} \quad \ldots \quad\left[D^{L-1}(C-E)^{\mathrm{T}}\right] \otimes \mathbf{R}_{L-1}^{\frac{1}{2}}}
\end{array}\right]
$$

$$
C=\left[\begin{array}{llllllllll}
D_{\mathbf{s}_{0}, 1} & D_{\mathbf{s}_{1}, 1} & \cdots & D_{\mathbf{s}_{G-1}, 1} & \cdots & \cdots & D_{\mathbf{s}_{0}, K} & D_{\mathbf{s}_{1}, K} & \cdots & D_{\mathbf{s}_{G-1}, K}
\end{array}\right]
$$




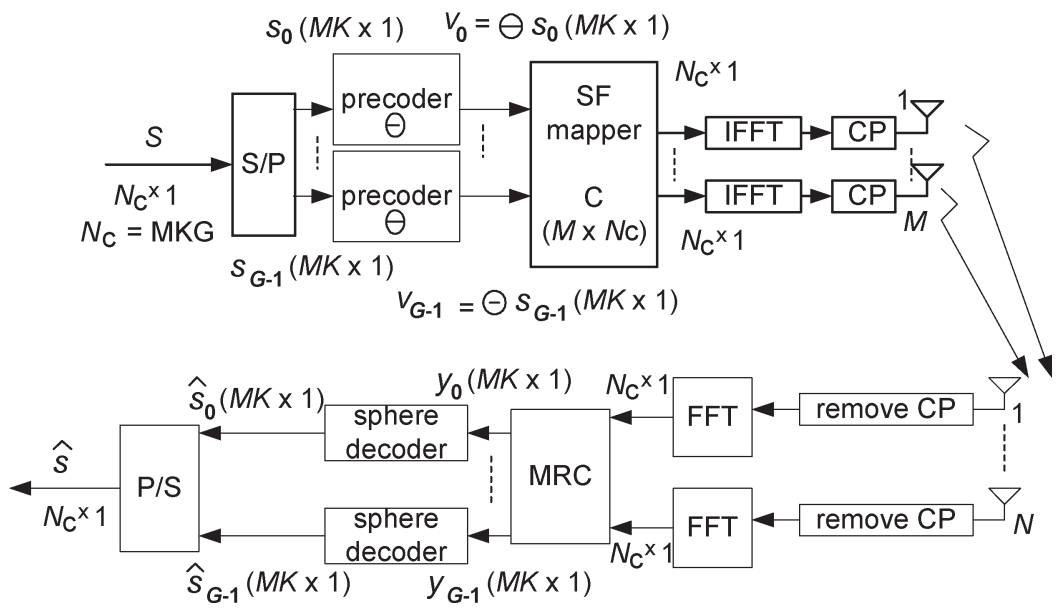

Fig. 1. Baseband multiple antenna OFDM system model.

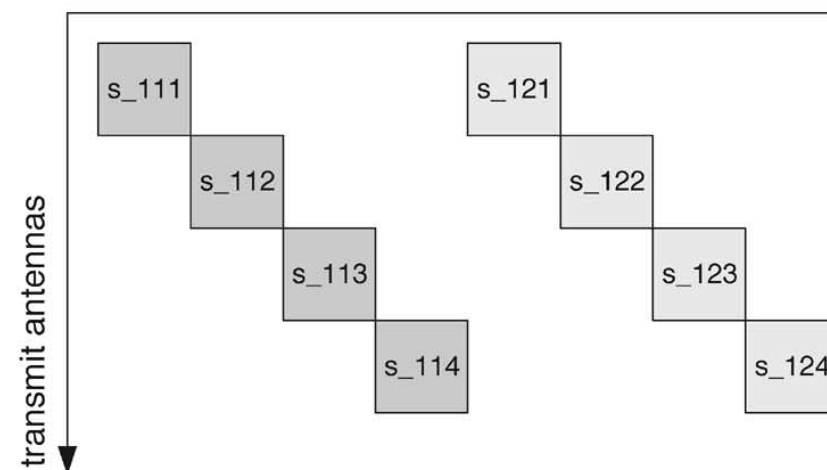

4 antennas, 16 subcarriers, 2 groups, 1 layer s_ijk = the $k$ th precoded symbol in the $j$ th group of the ith layer

Fig. 2. Structure of rate-one SFBC matrix C.

sufficiently large); however, because all the diversity is collected within each subgroup of subcarriers (as shown later in the proof), code performance will not be affected by the specific value of $G$. Further, we remark that if the maximum diversity order is not deemed necessary, we may choose $K<L$ in order to trade diversity (the achieved diversity order is $M N K$ in this case) for lower decoding complexity, as will be demonstrated.

\section{B. Precoder Design}

The general form of square CR matrices [7], [8] has row-wise Vandermonde structure

$$
\Theta=\frac{1}{\psi}\left[\begin{array}{cccc}
1 & \alpha_{0} & \cdots & \alpha_{0}^{M K-1} \\
1 & \alpha_{1} & \cdots & \alpha_{1}^{M K-1} \\
\vdots & \vdots & & \vdots \\
1 & \alpha_{M K-1} & \cdots & \alpha_{M K-1}^{M K-1}
\end{array}\right]
$$

where $\psi$ is a normalizing scalar that enforces the power constraint $E\left(\|\Theta \mathbf{s}\|^{2}\right)=E\left(\|\mathbf{s}\|^{2}\right)=M K$. It is shown in [8] that the $\mathrm{CR}$ precoder $\Theta$ achieves maximum diversity over any quadratic-amplitude modulation (QAM) constellation provided any of the following holds.

1) $M K$ is an Euler number, i.e., $M K \in \mathcal{S}_{1}=\{\phi(P)$ : $P \neq 0(\bmod 4)\}$, and $\left\{\alpha_{k}\right\}_{k=1}^{M K}$ are chosen to be roots of the equation $\Phi_{P}(x)=0$, where $\Phi_{P}(x)=\prod_{i \in \mathcal{I}}(x-$ $\left.\alpha^{i}\right)$ with $\mathcal{I}:=\{i \mid \operatorname{gcd}(i, P)=1$ and $i \in[1, p)\}$ and $\alpha:=$ $\exp [j 2 \pi / P]$.

2) $M K$ is a power of 2, i.e., $M K \in \mathcal{S}_{2}=\left\{2^{Q}: Q \in \mathbf{N}\right\}$, and $\left\{\alpha_{k}\right\}_{k=1}^{M K}$ are chosen as the roots of $x^{M K}-j=0$. The precoder in (5) becomes a unitary precoder that can be factored as: $\Theta=\mathbf{F}_{M K}^{\mathrm{T}} \operatorname{diag}\left(1, \alpha, \ldots, \alpha^{M K-1}\right)$, where $\mathbf{F}_{M K}$ is the $M K$-point inverse fast Fourier transform (IFFT) matrix with $(m+1, n+1)$ th en$\operatorname{try}(M K)^{-1 / 2} \exp [j 2 \pi m n /(M K)]$, and $\alpha:=\exp [j \pi /$ $(2 M K)]$.

3) $M K \notin \mathcal{S}_{1} \cup \mathcal{S}_{2}$, and $\left\{\alpha_{k}\right\}_{k=1}^{M K}$ are chosen to be the roots of $x^{M K}-(1+j)=0$.

In our SFBC, we make use of the following desirable property of $\Theta$ : for all distinct pairs $\left\{\mathbf{s}_{g}, \tilde{\mathbf{s}}_{g}\right\}$ and $\mathbf{v}_{g}=\Theta \mathbf{s}_{g}, \tilde{\mathbf{v}}_{g}=$ $\Theta \tilde{\mathbf{s}}_{g}$, the corresponding error vector $\mathbf{e}_{g}=\left(\mathbf{v}_{g}-\tilde{\mathbf{v}}_{g}\right)$ has all nonzero elements. Thus if we generate $D_{\tilde{\mathbf{s}}_{g}, k}, k=1, \ldots, K$ from $\tilde{\mathbf{s}}_{g}$, then the $K$ diagonal error matrices $\left(D_{\mathbf{s}_{g}, k}-D_{\tilde{\mathbf{s}}_{g}, k}\right)$ have all nonzero diagonal elements. Therefore, all distinct 
pairs $\left\{\mathbf{s}_{g}, \tilde{\mathbf{s}}_{g}\right\}$, give rise to $K$ full-rank diagonal error matrices $\left(D_{\mathbf{s}_{g}, k}-D_{\tilde{\mathbf{s}}_{g}, k}\right)$; this property will be used later in our proof.

\section{Diversity Order Evaluation}

Theorem 1: Under the assumption that $r\left(\mathbf{R}_{l}\right)=N$ for $l=$ $0,1, \ldots, L-1$, the SF codes proposed can achieve the diversity order of $M N L$ (or $M N K)$ for $K \geq L($ or $K<L)$, or equivalently, the diversity order is $\min (L, K) M N$.

Proof: As already argued based on the results in [25], it suffices to show that the theorem holds for the case of no spatial fading correlation with a uniform power delay profile. From (4), this is equivalent to proving that over all distinct $\{C, E\}$ pairs, $\min _{\{C, E\}} \operatorname{rank}(S)=M \min (L, K)$. Since $S=$ $G(C, E) G^{\mathrm{H}}(C, E)$ and $\operatorname{rank}(S)=\operatorname{rank}[G(C, E)]=\operatorname{rank}[G(C$, $\left.E)^{\mathrm{T}}\right]$, this is equivalent to showing that $\min _{\{C, E\}} \operatorname{rank}[G(C$, $\left.E)^{\mathrm{T}}\right]=M \min (L, K)$, where $G(C, E)^{\mathrm{T}}$ is an $M L \times N_{\mathrm{c}}$ matrix

$$
G(C, E)^{\mathrm{T}}=\left[\begin{array}{c}
(C-E) \\
(C-E) D \\
\vdots \\
(C-E) D^{L-1}
\end{array}\right]
$$

and $D=\operatorname{diag}\left\{\exp \left[-j\left(2 \pi / N_{\mathrm{c}}\right) k\right]\right\}_{k=0}^{N_{\mathrm{c}}-1}$.

As defined in Section II-A, $C=\left[\begin{array}{llll}D_{\mathbf{s}_{0}, 1} & D_{\mathbf{s}_{1}, 1} & \ldots\end{array}\right.$ $\left.\begin{array}{lllllll}D_{\mathbf{s}_{G-1}, 1} & \cdots & \cdots & D_{\mathbf{s}_{0}, K} & D_{\mathbf{s}_{1}, K} & \cdots & D_{\mathbf{s}_{G-1}, K}\end{array}\right]$, where $D_{\mathbf{s}_{g}, k}=\operatorname{diag}\left\{\boldsymbol{\theta}_{M \times(k-1)+1}^{\mathrm{T}} \mathbf{s}_{g} \cdots \boldsymbol{\theta}_{M \times k}^{\mathrm{T}} \mathbf{s}_{g}\right\}$, for $k=1, \ldots$, $K$. Similarly, let $E=\left[\begin{array}{llllll}D_{\tilde{\mathbf{s}}_{0}, 1} & D_{\tilde{\mathbf{s}}_{1}, 1} & \cdots & D_{\tilde{\mathbf{s}}_{G-1}, 1} & \cdots & \cdots\end{array}\right.$ $\left.\begin{array}{llll}D_{\tilde{\mathbf{s}}_{0}, K} & D_{\tilde{\mathbf{s}}_{1}, K} & \cdots & D_{\tilde{\mathbf{s}}_{G-1}, K}\end{array}\right]$. Consider the $N_{\mathrm{c}} \times 1$ vectors $\mathbf{s}=\left[\begin{array}{llll}\mathbf{s}_{0}^{\mathrm{T}} & \mathbf{s}_{1}^{\mathrm{T}} & \cdots & \mathbf{s}_{G-1}^{\mathrm{T}}\end{array}\right]^{\mathrm{T}}$, and $\tilde{\mathbf{s}}=\left[\begin{array}{llll}\tilde{\mathbf{s}}_{0}^{\mathrm{T}} & \tilde{\mathbf{s}}_{1}^{\mathrm{T}} & \cdots & \tilde{\mathbf{s}}_{G-1}^{\mathrm{T}}\end{array}\right]^{\mathrm{T}}$ such that $\mathbf{s} \neq \tilde{\mathbf{s}}$; then there exists $\mathbf{s}_{g} \neq \tilde{\mathbf{s}}_{g}$ for some $g \in$ $\{0, \ldots, G-1\}$. For rate-one SFBC, the $\min _{\{C, E\}} \operatorname{rank}[G(C$, $\left.E)^{\mathrm{T}}\right]$ is achieved when there is only one subvector $\mathbf{s}_{g} \neq \tilde{\mathbf{s}}_{g}$ for some $g$. Without loss of generality, let this be $\mathbf{s}_{0} \neq \tilde{\mathbf{s}}_{0}$.

Define the diagonal $M \times M$ matrices $A_{(k-1) G+g+1}=$ $D_{\mathbf{s}_{g}, k}-D_{\tilde{\mathbf{s}}_{g}, k}, k=1, \ldots, K ; g=0, \ldots, G-1$. Note that due to the property of the CR matrix $\Theta$, each of $\left\{A_{(k-1) G+g+1}\right\}$ will either be a full-rank (when $\mathbf{s}_{g} \neq \tilde{\mathbf{s}}_{g}$ ) or a zero matrix (when $\mathbf{s}_{g}=\tilde{\mathbf{s}}_{g}$ ). Thus, we can write $C-E=\left[\begin{array}{lll}A_{1} & \cdots & A_{G K}\end{array}\right]$.

We now partition the diagonal matrix $D=$ $\operatorname{diag}\left\{\exp \left[-j\left(2 \pi / N_{\mathrm{c}}\right) k\right]\right\}_{k=0}^{N_{\mathrm{c}}-1}$ into $G K \quad M \times M$ diagonal submatrices $\left\{D_{i}\right\}_{i=1}^{G K}$ such that $D=\operatorname{diag}\left\{\begin{array}{lll}D_{1} & \cdots & D_{G K}\end{array}\right\}$. It is clear that $D_{i} \neq D_{j} \neq 0, \forall i \neq j$ and $\forall i, \operatorname{rank}\left(D_{i}\right)=M$. In addition, it is easy to see that $D^{i}=\operatorname{diag}\left\{\begin{array}{lll}D_{1}^{i} & \cdots & D_{G K}^{i}\end{array}\right\}$ using property of block diagonal matrices.

Thus, we get

$$
\begin{aligned}
(C-E) D^{i} & =\left[\begin{array}{lll}
A_{1} & \cdots & A_{G K}
\end{array}\right]\left[\begin{array}{ccc}
D_{1}^{i} & 0 & \cdots \\
0 & \ddots & 0 \\
0 & \cdots & D_{G K}^{i}
\end{array}\right] \\
& =\left[\begin{array}{lll}
A_{1} D_{1}^{i} & \cdots & A_{G K} D_{G K}^{i}
\end{array}\right] \\
& =\left[\begin{array}{lll}
D_{1}^{i} A_{1} & \cdots & D_{G K}^{i} A_{G K}
\end{array}\right] \\
& =\left[\begin{array}{lll}
D_{1}^{i} & \cdots & D_{G K}^{i}
\end{array}\right]\left[\begin{array}{ccc}
A_{1} & 0 & \cdots \\
0 & \ddots & 0 \\
0 & \cdots & A_{G K}
\end{array}\right]
\end{aligned}
$$

where we used the fact that both $A_{j}$ and $D_{j}^{i}$ are diagonal matrices in the final step. Therefore

$$
\begin{aligned}
& G(C, E)^{\mathrm{T}}=\left[\begin{array}{cccc}
I_{M} & I_{M} & \cdots & I_{M} \\
D_{1} & D_{2} & \cdots & D_{G K} \\
\vdots & \vdots & \vdots & \vdots \\
D_{1}^{L-1} & D_{2}^{L-1} & \cdots & D_{G K}^{L-1}
\end{array}\right] \\
& \times\left[\begin{array}{ccc}
A_{1} & 0 & \cdots \\
0 & \ddots & 0 \\
0 & \cdots & A_{G K}
\end{array}\right] .
\end{aligned}
$$

The first matrix on the right-hand side is of size $M L \times N_{\mathrm{c}}$ (for $N_{\mathrm{c}}=M G K$ ) and the second matrix is of size $N_{\mathrm{c}} \times N_{\mathrm{c}}$. Note that the second matrix is not always full rank.

Define $P=\min (L, K)$. As previously shown that over all distinct pairs $\left\{\mathbf{s}_{0}, \tilde{\mathbf{s}}_{0}\right\}$, we have $K$ full-rank diagonal error matrices $A_{(k-1) G+1}=\left(D_{\mathbf{s}_{0}, k}-D_{\tilde{\mathbf{s}}_{0}, k}\right)$, for $k=1, \ldots, K$. Thus, in $G(C, E)^{\mathrm{T}}$, we can find an $M P \times M P$ submatrix which is the product of two other $M P \times M P$ matrices as follows:

$$
\begin{aligned}
& {\left[\begin{array}{cccc}
I_{M} & I_{M} & \cdots & I_{M} \\
D_{1} & D_{G+1} & \cdots & D_{(P-1) G+1} \\
\vdots & \vdots & \vdots & \vdots \\
D_{1}^{P}-1 & D_{G+1}^{P-1} & \cdots & D_{(P-1) G+1}^{P-1}
\end{array}\right] } \\
& \\
& \times\left[\begin{array}{cccc}
A_{1} & 0 & & \\
0 & A_{G+1} & & \cdots \\
0 & & \ddots & 0 \\
0 & \cdots & & A_{(P-1) G+1}
\end{array}\right] .
\end{aligned}
$$

The first submatrix is block Vandermonde and composed of diagonal submatrices and it is easy to prove that it has a nonzero determinant. The second submatrix is a block diagonal matrix composed of full-rank diagonal submatrices and thus has a nonzero determinant. Therefore, the product also has a nonzero determinant and is of full rank $M P=M \min (L, K)$. Since $G(C, E)^{\mathrm{T}}\left(\operatorname{dim} M L \times N_{\mathrm{c}}, M L<N_{\mathrm{c}}\right)$ has rank at most $M L$ and we can always find a submatrix of dimension $M P \times M P$ which is of full rank, the minimum rank achieved is $M P$ [note in this case $G(C, E)$ may not be full rank]. Thus, we conclude that $\min _{\{\mathbf{s}, \tilde{\mathbf{s}}\}} \operatorname{rank}\left[G(C, E)^{\mathrm{T}}\right]=M P=M \min (L, K)$. Because diversity order is determined by the minimum rank of $G(C, E)^{\mathrm{T}}$ over all distinct $\{\mathbf{s}, \tilde{\mathbf{s}}\}$ pairs (equiv. all distinct $\{C, E\}$ pairs), we conclude that the diversity achieved by our SFBC is of order $M N \min (L, K)$, which completes our proof.

Comment: We propose a suboptimal but near maximum likelihood decoding scheme in the next section, whose complexity is polynomial in the OFDM symbol length. If the maximum diversity order is not deemed necessary, we may choose $K<L$ in order to trade maximum possible diversity order $M N K$ for lower decoding complexity.

\section{Coding Gain Evaluation}

Following [23] and [24], the idea of subchannel grouping is applied in code construction. Once again we restrict our attention to the special case of no spatial fading correlation with 
uniform power delay profile, for receive correlation essentially results in loss of coding gain that is independent of the code design [25]. In order to evaluate coding gain, we rewrite (4) based on subchannel grouping as follows:

For the $g$ th group

$$
P\left(C_{g} \rightarrow E_{g}\right) \leq \prod_{i=0}^{\operatorname{rank}\left(S_{g}\right)-1}\left[1+\lambda_{i}\left(S_{g}\right) \frac{\rho}{4}\right]^{-N}
$$

where $S_{g}=G_{g}\left(C_{g}, E_{g}\right) G_{g}\left(C_{g}, E_{g}\right)^{\mathrm{H}}$ has dimension $M K \times$ $M K$ and $\lambda_{i}\left(S_{g}\right)$ is the $i$ th nonzero eigenvalue of $S_{g}$. $G_{g}\left(C_{g}, E_{g}\right)$ is an $M K \times M L$ matrix defined as

$$
\begin{aligned}
& G_{g}\left(C_{g}, E_{g}\right) \\
& =\left[\begin{array}{llll}
\left(C_{g}-E_{g}\right)^{\mathrm{T}} & D_{\text {sub }, g}\left(C_{g}-E_{g}\right)^{\mathrm{T}} & \cdots & D_{\text {sub }, g}^{L-1}\left(C_{g}-E_{g}\right)^{\mathrm{T}}
\end{array}\right]
\end{aligned}
$$

and $D_{\text {sub }, g}=\operatorname{diag}\left\{\begin{array}{llll}D_{g} & D_{G+g} & \cdots & D_{(K-1) G+g}\end{array}\right.$ is composed of the diagonal submatrices $\left\{D_{i}\right\}$ as defined in proof of Theorem 1 and $C_{g}=\left[\begin{array}{llll}D_{\mathbf{s}_{g}, 1} & D_{\mathbf{s}_{g}, 2} & \cdots & D_{\mathbf{s}_{g}, K}\end{array}\right]$ with $E_{g}$ defined similarly. Thus, $C_{g}-E_{g}=\left[\begin{array}{lll}A_{g} A_{G+g} & \cdots & A_{(K-1) G+g}\end{array}\right]$ using the definition of $A_{i}$ in proof of Theorem 1.

Therefore, the coding gain for our SFBC based on subchannel grouping is

$$
G_{g, c}=\min _{\forall C_{g} \neq E_{g}}\left[\prod_{i=0}^{\operatorname{rank}\left(S_{g}\right)-1} \lambda_{i}\left(S_{g}\right)\right]^{\frac{1}{\operatorname{rank}\left(S_{g}\right)}} .
$$

Under the assumption of Theorem 1 for our SFBC, $\operatorname{rank}\left(S_{g}\right)=$ $M \min (L, K)$. In addition, the coding gain brought by LCF precoding is $\eta_{l c p}=\min _{\left\{\mathbf{s}_{g}, \tilde{\mathbf{s}}_{g}\right\}} \prod_{k=1}^{M K}\left|\theta_{k}^{\mathrm{T}}\left(\mathbf{s}_{g}-\tilde{\mathbf{s}}_{g}\right)\right|^{2}=$ $\left(\Delta_{\min }^{2} / \beta^{2}\right)^{M K}$, where $\Delta_{\min }$ is the minimum distance among signal points and $\beta^{2}=M K$ if $M K$ is an Euler number or a power of two; otherwise, $\beta^{2}=1 /\left(2^{(1 / M K)}-1\right)$ [23]. The coding gain of LCF precoder is maximized if $M K$ is an Euler number or a power of two [7], [8]. In the following, we give the main results of coding gain evaluation and leave the derivation in the Appendix.

Case I: $K<L$ : The coding gain is

$$
\begin{array}{r}
G_{g, c}=\sqrt[K]{\prod_{n=0}^{K-1}\left[\left(\left\lfloor\frac{L-1-n}{K}\right\rfloor+1\right) K\right]} \\
\times\left(\frac{\Delta_{\min }^{2}}{\beta^{2}}\right) \leq L \times\left(\frac{\Delta_{\min }^{2}}{\beta^{2}}\right)
\end{array}
$$

where $\lfloor x\rfloor$ is the largest integer contained in $x$. The equality holds only when $L=m K, m=2,3, \ldots$. From the right-hand side of (13), we find that the coding gain of SFBC is affected by two factors: i) the first term is due to symbol mapping and ii) the second term is determined by LCF precoding. The detailed derivation of coding gain is in the Appendix.
Case II: $K>L$

$$
G_{g, c}=\min _{\forall C_{g} \neq E_{g}} K \times\left(\prod_{j=1}^{M L} \beta_{j}\right)^{\frac{1}{M L}}
$$

where $0<\min _{k \in 1, \ldots, M K}\left|\theta_{k}^{\mathrm{T}}\left(\mathbf{s}_{g}-\tilde{\mathbf{s}}_{g}\right)\right|^{2} \leq \beta_{j} \leq$ $\max _{k \in 1, \ldots, M K}\left|\theta_{k}^{\mathrm{T}}\left(\mathbf{s}_{g}-\tilde{\mathbf{s}}_{g}\right)\right|^{2}$. The derivation of coding gain is in the Appendix.

Case III: $K=L$ : The coding gain is

$$
G_{g, c}=L \times \frac{\Delta_{\min }^{2}}{\beta^{2}}
$$

where $\beta^{2}=M L$ if $M L$ is an Euler number or a power of two; otherwise, $\beta^{2}=1 /\left(2^{(1 / M L)}-1\right)$ [23]. The two terms on the right-hand side have the same significance as in Case I. The derivation can be found in the Appendix.

In [20], using a more generalized channel model, the maximum coding gain for these linearly coded systems was investigated. Assuming that maximum diversity is achieved and that channel correlation matrix $\mathbf{R}_{h}$ has full rank, the maximum coding gain [20] corresponding to the maximum diversity gain of $M N L$ was shown to be $G_{\mathrm{c}}^{\max }=\left[\operatorname{det}\left(\mathbf{R}_{h}\right)\right]^{1 / M N L}\left(\Delta_{\min }^{2} / M\right)$. Since we use the same precoder as in [20], we can make a fair comparison between the coding gain achieved by our SFBC and the maximum coding gain (both corresponding to the maximum diversity $M N L$ ) for the case of no spatial correlation (i.e., $\mathbf{R}_{h}=I_{M N L}$ ). We define the relative coding gain as the ratio

$$
G_{g, c, \text { relative }}=\frac{L \frac{\Delta_{\min }^{2}}{\beta^{2}}}{\frac{\Delta_{\min }^{2}}{M}}=L \frac{M}{\beta^{2}} .
$$

Because the optimal LCF precoder proposed by [7] and [8] ensures that for the case of $K=L$ and $M L$ is an Euler number or a power of two, $\beta^{2}=M L$, the above equation simplifies to

$$
G_{g, c, \text { relative }}=L \frac{1}{L}=1 .
$$

Therefore, under the condition that $M L$ is an Euler number or a power of two (i.e., the precoder's $\eta_{l c p}$ is maximized), our SFBC indeed achieves maximum coding gain among linearly coded systems, i.e., our symbol mapping pattern is optimal. When $M L$ takes other values (where $\eta_{l c p}$ is not maximized), the achieved relative coding gain is around $0.7-0.78$ and decreases as $M L$ increases.

\section{Space-Frequency Decoding}

From Fig. 1, for each individual OFDM block time, the received signal at the $j$ th receive antenna can be expressed as

$r_{j}[l]=\sum_{i=1}^{M} H_{i j}[l] t_{i}[l]+w_{j}[l], \quad l=1, \ldots, N_{\mathrm{c}}, j=1, \ldots, N$ 
where $H_{i j}[l]$ denotes the channel frequency response of the $i$ th transmit and $j$ th receive antenna pair at the $l$ th tone, $t_{i}[l]$ denotes the transmitted signal from the $i$ th transmit antenna at the $l$ th tone. In addition, $r_{j}[l]$ and $w_{j}[l]$ denote the received signal and additive white Gaussian noise component at the $j$ th receive antenna for the $l$ th tone, respectively.

Let $\mathbf{r}^{j}, \mathbf{w}^{j}$ denote the size $N_{\mathrm{c}} \times 1$ received signal vector and noise vector at the $j$ th receive antenna, respectively. Now $\mathbf{r}^{j}$ can be partitioned into $K G$ size $M \times 1$ subvectors $\left\{\mathbf{r}^{j, k, g}\right\}$ for $g, k$ with the same definition as that in Section II-A. Similarly, $\mathbf{w}^{j}$ can be subdivided into $K G$ size $M \times 1$ subvectors $\left\{\mathbf{w}^{j, k, g}\right\}$. Therefore

$$
\begin{aligned}
\mathbf{r}^{j, k, g}= & {\left[\begin{array}{c}
r_{j}[(k-1) G M+g M] \\
\vdots \\
r_{j}[(k-1) G M+(g+1) M-1]
\end{array}\right] } \\
\mathbf{w}^{j, k, g}= & {\left[\begin{array}{c}
w_{j}[(k-1) G M+g M] \\
\vdots \\
w_{j}[(k-1) G M+(g+1) M-1]
\end{array}\right] . }
\end{aligned}
$$

In addition, $\boldsymbol{\theta}_{i}^{\mathrm{T}}$ denotes the $i$ th row of the CR matrix $\Theta$ and $H_{i, l}^{j}=H_{i, j}[l]$. Also, we define the diagonal matrix

$$
\Lambda_{k, g}^{j}=\operatorname{diag}\left\{\begin{array}{c}
H_{1,(k-1) G M+g M}^{j} \\
H_{2,(k-1) G M+g M+1}^{j} \\
\vdots \\
H_{M,(k-1) G M+(g+1) M-1}^{j}
\end{array}\right\} \text {. }
$$

Therefore, for our SFBC, we have

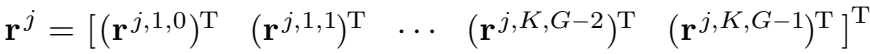

$$
\begin{aligned}
& =\left[\begin{array}{l}
\mathbf{b}^{j, 1,0} \\
\mathbf{b}^{j, 1,1} \\
\vdots \\
\mathbf{b}^{j, K, G-2} \\
\mathbf{b}^{j, K, G-1}
\end{array}\right]+\left[\begin{array}{l}
\mathbf{w}^{j, 1,0} \\
\mathbf{w}^{j, 1,1} \\
\vdots \\
\mathbf{w}^{j, K, G-2} \\
\mathbf{w}^{j, K, G-1}
\end{array}\right]
\end{aligned}
$$

where

$$
\begin{aligned}
\mathbf{b}^{j, k, g}= & {\left[\begin{array}{l}
H_{1,(k-1) G M+g M}^{j} \boldsymbol{\theta}_{(k-1) M+1}^{\mathrm{T}} \mathbf{s}_{g} \\
H_{2,(k-1) G M+g M+1}^{j} \boldsymbol{\theta}_{(k-1) M+2}^{\mathrm{T}} \mathbf{s}_{g} \\
\vdots \\
H_{M,(k-1) G M+(g+1) M-1}^{j} \boldsymbol{\theta}_{k M}^{\mathrm{T}} \mathbf{s}_{g}
\end{array}\right] } \\
= & \Lambda_{k, g}^{j}\left[\begin{array}{l}
\theta_{(k-1) M+1}^{\mathrm{T}} \\
\theta_{(k-1) M+2}^{\mathrm{T}} \\
\vdots \\
\theta_{k M}^{\mathrm{T}}
\end{array}\right] \mathbf{s}_{g}
\end{aligned}
$$

and $\mathbf{r}^{j, k, g}=\mathbf{b}^{j, k, g}+\mathbf{w}^{j, k, g}$.
Combining those sub-blocks in the $g$ th group, we get

$$
\begin{aligned}
& \mathbf{r}^{j, g}=\left[\begin{array}{llll}
\mathbf{r}^{j, 1, g} & \mathbf{r}^{j, 2, g} & \cdots & \mathbf{r}^{j, K, g}
\end{array}\right]^{\mathrm{T}} \\
& =\left[\begin{array}{l}
\mathbf{b}^{j, 1, g} \\
\mathbf{b}^{j, 2, g} \\
\vdots \\
\mathbf{b}^{j, K, g}
\end{array}\right]+\underbrace{\left[\begin{array}{l}
\mathbf{w}^{j, 1, g} \\
\mathbf{w}^{j, 2, g} \\
\vdots \\
\mathbf{w}^{j, K, g}
\end{array}\right]}_{\mathbf{w}^{j, g}} \\
& =\underbrace{\left[\begin{array}{ccc}
\Lambda_{1, g}^{j} & & \\
& \ddots & \\
& & \Lambda_{K, g}^{j}
\end{array}\right]}_{\Lambda_{g}^{j}} \Theta \mathbf{s}_{g}+\mathbf{w}^{j, g} .
\end{aligned}
$$

Furthermore, we can combine information from the $g$ th group over the $N$ receive antennas to get

$$
\underbrace{\left[\begin{array}{l}
\mathbf{r}^{1, g} \\
\mathbf{r}^{2, g} \\
\vdots \\
\mathbf{r}^{N, g}
\end{array}\right]}_{\mathbf{r}_{g}}=\underbrace{\left[\begin{array}{l}
\Lambda_{g}^{1} \\
\Lambda_{g}^{2} \\
\vdots \\
\Lambda_{g}^{N}
\end{array}\right]}_{\Lambda_{g}} \Theta \mathbf{s}_{g}+\underbrace{\left[\begin{array}{l}
\mathbf{w}^{1, g} \\
\mathbf{w}^{2, g} \\
\vdots \\
\mathbf{w}^{N, g}
\end{array}\right]}_{\mathbf{w}_{g}} .
$$

Left multiplying $\Lambda_{g}^{\mathrm{H}}$ to both sides of (25), we obtain

$\Lambda_{g}^{\mathrm{H}} \mathbf{r}_{g}=\left[\begin{array}{lll}\left(\Lambda_{g}^{1}\right)^{\mathrm{H}} & \cdots & \left(\Lambda_{g}^{N}\right)^{\mathrm{H}}\end{array}\right]\left[\begin{array}{l}\Lambda_{g}^{1} \\ \vdots \\ \Lambda_{g}^{N}\end{array}\right] \Theta \mathbf{s}_{g}+\underbrace{\Lambda_{g}^{\mathrm{H}} \mathbf{w}_{g}}_{\mathbf{n}_{g}}$

$$
=\left[\sum_{j=1}^{N}\left(\Lambda_{g}^{j}\right)^{\mathrm{H}} \Lambda_{g}^{j}\right] \Theta \mathbf{s}_{g}+\mathbf{n}_{g}
$$

$$
=\underbrace{\left[\begin{array}{lll}
\sum_{j=1}^{N}\left(\Lambda_{1, g}^{j}\right)^{\mathrm{H}} \Lambda_{1, g}^{j} & & \\
& \ddots & \\
& & \sum_{j=1}^{N}\left(\Lambda_{K, g}^{j}\right)^{\mathrm{H}} \Lambda_{K, g}^{j}
\end{array}\right]}_{\Sigma_{g}}
$$

$$
\times \Theta \mathbf{s}_{g}+\mathbf{n}_{g} .
$$

Since $\mathbf{n}_{g}$ is no longer white Gaussian, it is prewhitened prior to decoding.

$$
\begin{aligned}
\mathbf{y}_{g} & =\Sigma_{g}^{-\frac{1}{2}} \Lambda_{g}^{\mathrm{H}} \mathbf{r}_{g} \\
& =\Sigma_{g}^{\frac{1}{2}} \Theta \mathbf{s}_{g}+\boldsymbol{\eta}_{g}
\end{aligned}
$$

where $\boldsymbol{\eta}_{g}=\Sigma_{g}^{-1 / 2} \mathbf{n}_{g}$ denotes the whitened Gaussian noise.

Finally, a sphere decoder [11], [17], which can achieve nearoptimum performance (in the maximum likelihood sense) with 


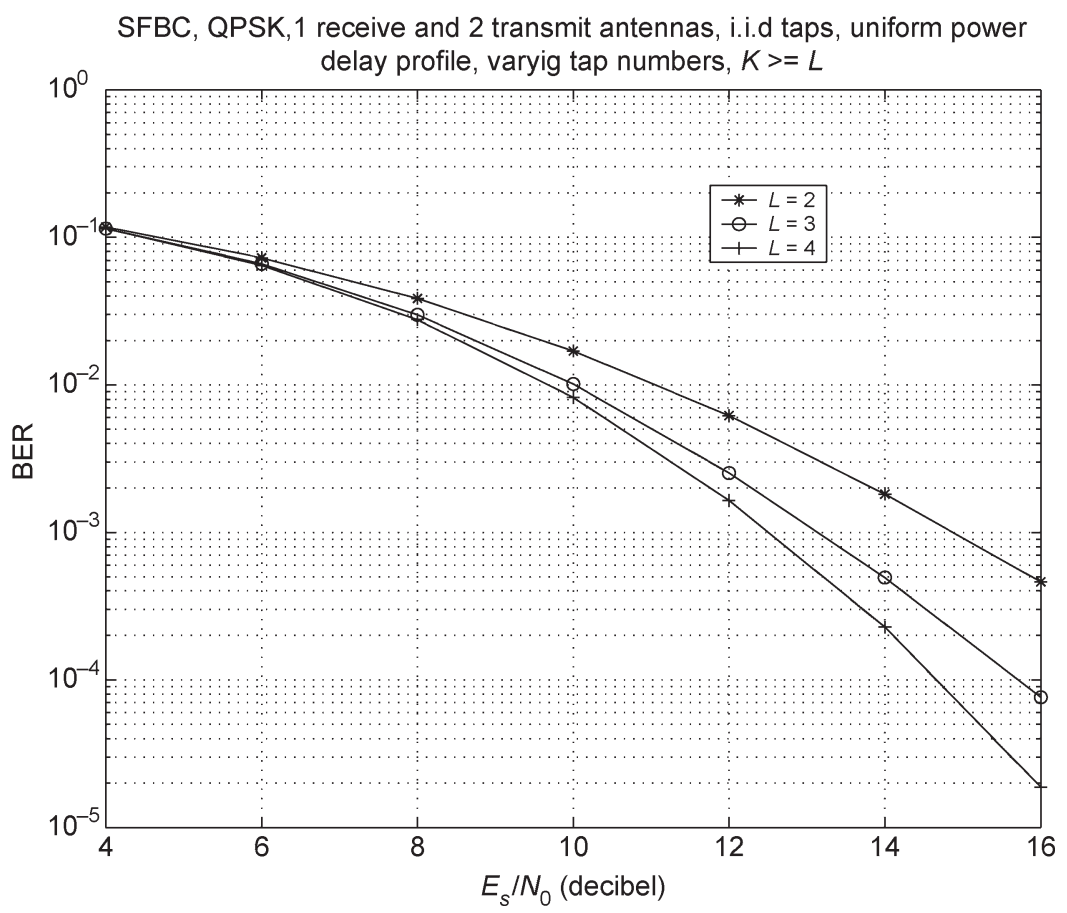

Fig. 3. BER performance of SFBC with different channel taps $(M=2, N=1)$.

average polynomial complexity in $M K$ [e.g., $O(2 M K)^{3}$ for complex value $\Theta$ ] regardless of the constellation size, is used to decode $\mathbf{s}_{g}$ from $\mathbf{y}_{g}$.

\section{Simulation Results}

In the simulations, we consider an OFDM system with parameters conforming to the IEEE 802.16.3 standard. The FFT size is 256 and the number of subcarriers used is 200 (including 8 for pilots and 192 for data). The one-sided radio frequency bandwidth assumed in the simulation is $6 \mathrm{MHz}$ with a sampling rate of $7 \mathrm{MHz}$. The carrier frequency spacing $\Delta_{f}$ is $0.02734 \mathrm{MHz}$. The data symbol duration is $T_{u}=36.57 \mu \mathrm{s}$ and the $\mathrm{CP}$ duration is $T_{g}=9.14 \mu \mathrm{s}$ for total symbol duration $T_{\text {total }}=45.71 \mu \mathrm{s} .16$-QAM with code rate $3 / 4$ achieves a data rate of $12.6 \mathrm{Mb} / \mathrm{s}$; thus, our rate-one SFBC-OFDM system would achieve a data rate of $16.8 \mathrm{Mb} / \mathrm{s}$. Modulation symbols used were binary phase-shift keying (BPSK), 4-QAM or 16-QAM where the total average symbol energy on all $M$ transmit antennas were normalized $E_{\mathrm{S}}=1$. The variance $\sigma^{2}$ of the white Gaussian noise per dimension is adjusted by the formula $\sigma^{2}=(1 / 2) 10^{-(\mathrm{SNR} / 10)}$. The $L$-tap frequency-selective channel for each transmit-receive antenna pair has independent and identically distributed complex Gaussian coefficients with total power $=1.10^{5}$ independent channel realizations (each channel realization corresponds to one data packet which consists of one OFDM symbol block for simplicity) were used to obtain the simulation results. In Fig. 3, we assume that $N_{\mathrm{c}}=M \times G \times L$ where $N_{\mathrm{c}}, L, M, G$ denote the number of tones, the length of the channel impulse response (less than or equal to the $\mathrm{CP}$ ), the number of transmit antennas, and the number of groups the tones are divided into, respectively. For a given $M$, performance is unaffected by the choice of $G$ since the code design ensures that all the diversity is actually achieved within each group of subcarriers. It is clear from the results that in $L$-tap frequency-selective fading channel, the diversity achieved increases with $L$ increase.

We then compare space-frequency block code-OFDM (SFBC-OFDM) with circular generalized delay diversityOFDM (CGDD-OFDM) proposed in [19] and Bell Labs Layered Space-Time-OFDM (BLAST-OFDM) [16] when ML detection is used for all the three schemes. The performance of the 802.11a OFDM standard with 16-QAM signaling and rate $1 / 2$ convolutional code using one transmit and receive antenna and deinterleaving cum Viterbi decoding is also included to establish a performance baseline and indicate the extent of additional gains that are achievable. In the simulations, the number of subcarriers was 64 and two transmit and receive antennas each were used for BLAST-OFDM, SFBC-OFDM, and CGDD-OFDM. In order to preserve identical bit rates for a fair comparison, we chose BPSK for BLAST-OFDM, 16-QAM for convolutional coding + interleaving, while using quaternary phase-shift keying (QPSK) for CGDD-OFDM and SFBC-OFDM. From Fig. 4, we can see that SFBC-OFDM outperforms the other three schemes as only it achieves the maximum diversity of $M N L$.

For completeness, we also compared our method to the STFBC method proposed in [5] and DPS of [20] with two or four transmit antennas and one receive antenna. In order to keep the same bit rate when four transmit antennas are used, we chose QPSK for STF, while using BPSK for both DPS and SFBC. From Fig. 5, we find that although STFBC [5] can guarantee maximum coding gain among all STF codes, both DPS and SFBC have very similar performances; the performance gap between them is reduced as the number of transmit antennas increases. In addition, although the code 


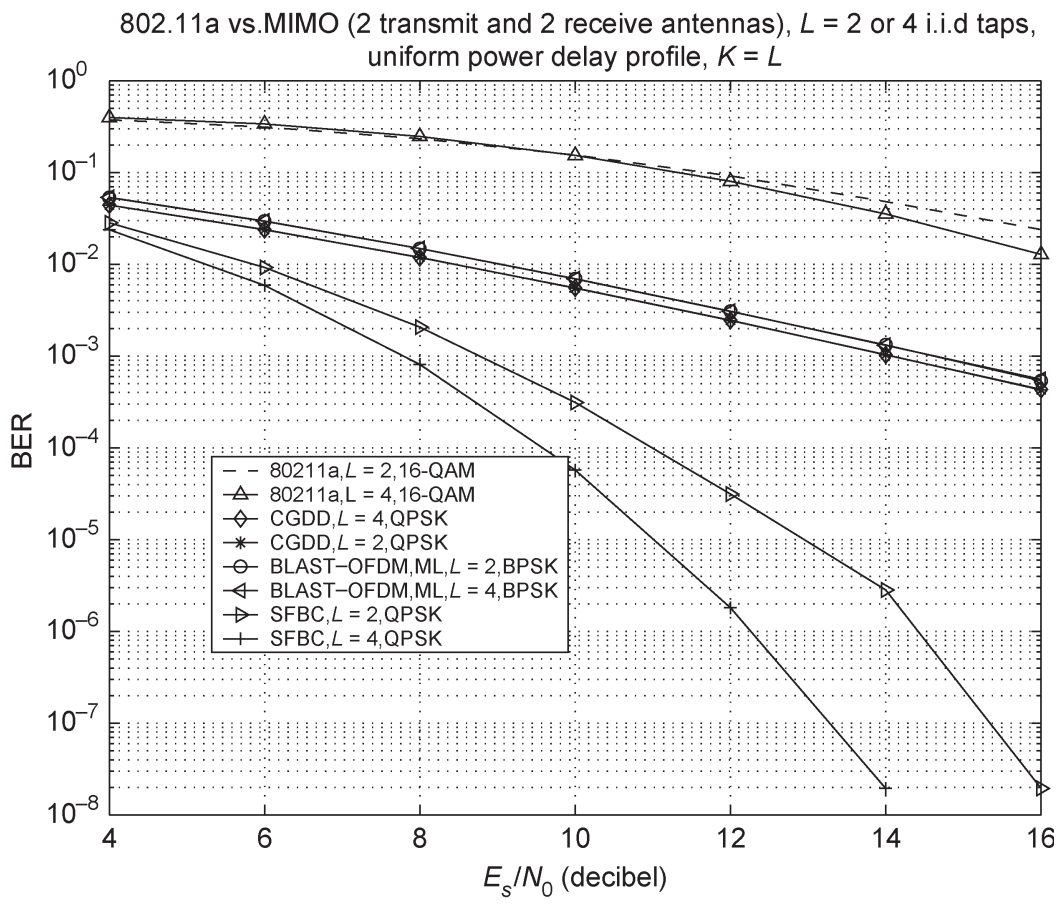

Fig. 4. BER performance comparison among CGDD-OFDM, BLAST-OFDM, SFBC-OFDM with ML detection ( $M=2, N=2,64$ subcarriers).

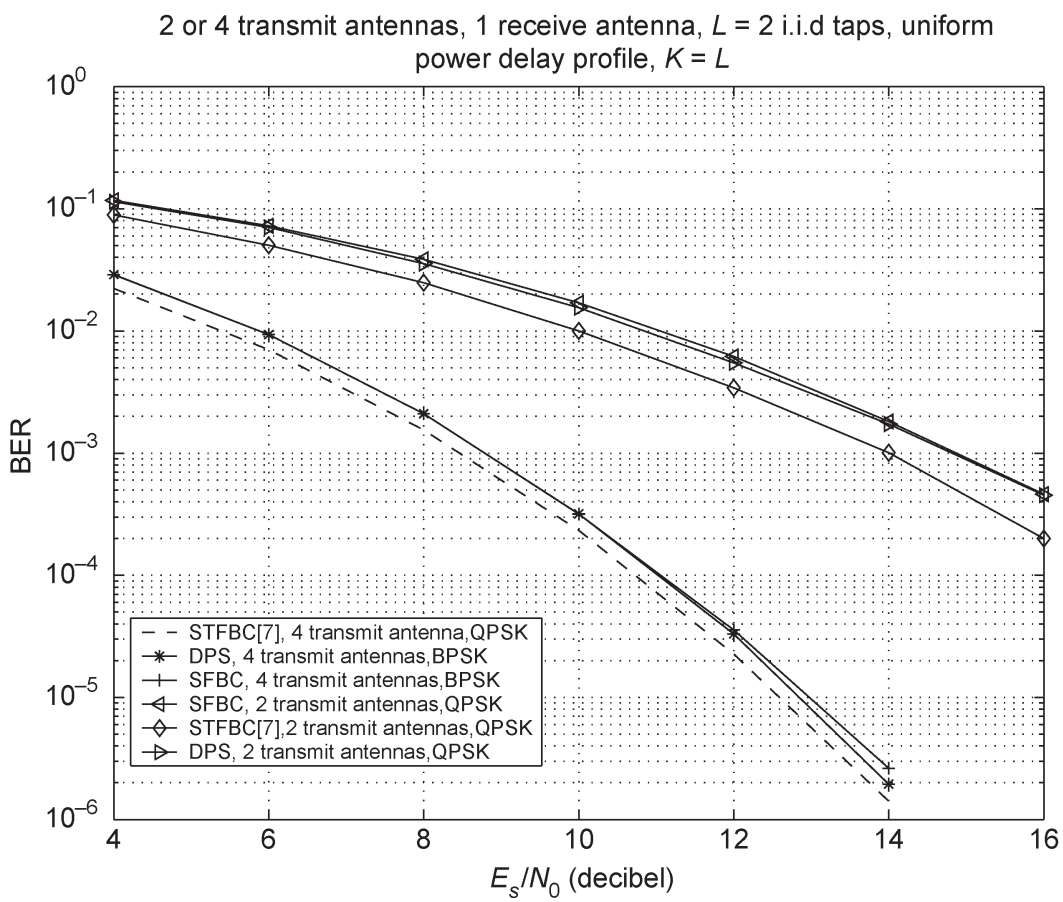

Fig. 5. BER performance comparison among STFBC [7], DPS [26] and proposed SFBC-OFDM.

matrices of DPS and SFBC are quite different, the bit error rate performances of the two are nearly the same. Since both of them use precoder of size $M L$, their decoding complexities are very similar as well.

\section{CONCLUSION}

In this paper, we proposed a novel rate-one SF code that achieves the maximum diversity over frequency-selective channels. Moreover, the code design shows robustness when the channel order is not known exactly at the transmitter and allows an easy tradeoff between diversity and decoding complexity. In addition, the processing delay is smaller compared to that of STFBC.

\section{APPENDIX}

A. $K<L$

For $K<L, \operatorname{rank}\left(S_{g}\right)=M K$. From (11), it follows that $G_{g} G_{g}^{\mathrm{H}}$ is a full-rank matrix with size $M K \times M K$, and since 
$\prod_{i=0}^{\operatorname{rank}\left(S_{g}\right)-1} \lambda_{i}\left(S_{g}\right)=\operatorname{det}\left[G_{g}\left(C_{g}, E_{g}\right) G_{g}\left(C_{g}, E_{g}\right)^{\mathrm{H}}\right]$, the coding gain is

$$
G_{g, c}=\min _{\forall C_{g} \neq E_{g}}\left\{\operatorname{det}\left[G_{g}\left(C_{g}, E_{g}\right) G_{g}\left(C_{g}, E_{g}\right)^{\mathrm{H}}\right]\right\}^{\frac{1}{M K}}
$$

since $G_{g} G_{g}^{\mathrm{H}}$ would yield the expression shown in (31) at the bottom of the page. Exploring the commutative property of diagonal matrices, we can rewrite $D_{\text {sub, } g}^{i}\left(C_{g}-E_{g}\right)^{\mathrm{T}}$ $\left(C_{g}-E_{g}\right)^{*} D_{\text {sub }, g}^{\mathrm{H}}$ as in (32) shown at the bottom of the page. Therefore

$$
G_{g} G_{g}^{\mathrm{H}}=\mathbf{B}_{g} \mathbf{W}_{1} \mathbf{W}_{1}^{\mathrm{H}} \mathbf{B}_{g}^{\mathrm{H}}
$$

where size $M K \times M L \mathbf{W}_{1}$ is defined as

$$
\mathbf{W}_{1}=\left[\begin{array}{cccc}
I_{M} & D_{g} & \cdots & D_{g}^{L-1} \\
I_{M} & D_{G+g} & \vdots & D_{G+g}^{L-1} \\
\vdots & & & \vdots \\
I_{M} & D_{(K-1) G+g} & \cdots & D_{(K-1) G+g}^{L-1}
\end{array}\right]
$$

Noting that both $\mathbf{B}_{g}$ and $\mathbf{W}_{1} \mathbf{W}_{1}^{\mathrm{H}}$ are of size $M K \times M K$ and using the property $\operatorname{det}(A B)=\operatorname{det}(A) \operatorname{det}(B)=\operatorname{det}(B A)$ for square matrices, we get

$$
\operatorname{det}\left(G_{g} G_{g}^{\mathrm{H}}\right)=\operatorname{det}\left(\mathbf{B}_{g}^{\mathrm{H}} \mathbf{B}_{g}\right) \operatorname{det}\left(\mathbf{W}_{1} \mathbf{W}_{1}^{\mathrm{H}}\right) .
$$

After some derivation, we arrive at (37) shown at the bottom of the page. The elements of the $K \times K$ matrix $\mathbf{J}$ defined above satisfy $a_{i, j}=a_{i+k, j+k}$ and $a_{i, j}=a_{j, i}^{*}, \forall i j, k$; hence matrix $\mathbf{J}$ is Hermitian Toeplitz. Moreover, $a_{m, K}=a_{m+1,1}$ $\forall m=1, \ldots, K$ since $\sum_{i=0}^{L-1} \exp (-j\{[2 \pi(K-m) i] / K\})=$ $\sum_{i=0}^{L-1} \exp [j(2 \pi m i / K)]$; hence matrix $\mathbf{J}$ is also circulant (Hermitian). Therefore, from a well-known result for the determinant of a circulant matrix, $\operatorname{det}(\mathbf{J})=\prod_{n=0}^{K-1} a\{\exp [j(2 \pi n /$ $K)]\}$ [21], where $a(x)=L+\sum_{k=1}^{K-1}\left\{\sum_{i=0}^{L-1} \exp [-j(2 \pi k i)\right.$ $K)]\} x^{k}$. Using the property that $\sum_{k=0}^{K-1} \exp [j(2 \pi k i / K)]=$ $0, \quad 1 \leq i \leq K-1$ and $\exp \{j[2 \pi k(l K) / K]\}=1$ for any integer $l$, it follows that $a\{\exp [j(2 \pi n / K)]\}=(\lfloor(L-1-$ $n) / K\rfloor+1) K, K<L$. Therefore, $\operatorname{det}(\mathbf{J})=\prod_{n=0}^{K-1}[(\lfloor(L-$ $1-n) / K\rfloor+1) K] \leq L^{K}$ where the equality holds only when

$$
G_{g} G_{g}^{\mathrm{H}}=\left[\begin{array}{llll}
\left(C_{g}-E_{g}\right)^{\mathrm{T}} & D_{\mathrm{sub}, g}\left(C_{g}-E_{g}\right)^{\mathrm{T}} & \cdots & D_{\mathrm{sub}, g}^{L-1}\left(C_{g}-E_{g}\right)^{\mathrm{T}}
\end{array}\right]\left[\begin{array}{c}
\left(C_{g}-E_{g}\right)^{*} \\
{\left[\begin{array}{c}
\left(C_{g}-E_{g}\right)^{*} D_{\mathrm{sub}, g}^{\mathrm{H}} \\
\vdots \\
\vdots \\
\left(C_{g}-E_{g}\right)^{*}\left(D_{\mathrm{sub}, g}^{L-1}\right)^{\mathrm{H}}
\end{array}\right]}
\end{array}\right]
$$

$$
\begin{aligned}
& D_{\text {sub }, g}^{i}\left(C_{g}-E_{g}\right)^{\mathrm{T}}\left(C_{g}-E_{g}\right)^{*} D_{\mathrm{sub}, \mathrm{g}}^{\mathrm{H}}=\underbrace{\left[\begin{array}{llll}
A_{g}^{\mathrm{T}} & & & \\
& A_{G+g}^{\mathrm{T}} & & \\
& & \ddots & \\
& & & A_{(K-1) G+g}^{\mathrm{T}}
\end{array}\right]}_{\mathbf{B}_{g}}\left[\begin{array}{c}
D_{g}^{i} \\
D_{G+g}^{i} \\
\vdots \\
D_{(K-1) G+g}^{i}
\end{array}\right] \\
& \times\left[\begin{array}{lll}
D_{g}^{-i} & \cdots & D_{(K-1) G+g}^{-i}
\end{array}\right]\left[\begin{array}{llll}
A_{g}^{*} & & & \\
& A_{G+g}^{*} & & \\
& & \ddots & \\
& & & A_{(K-1) G+g}^{*}
\end{array}\right]
\end{aligned}
$$

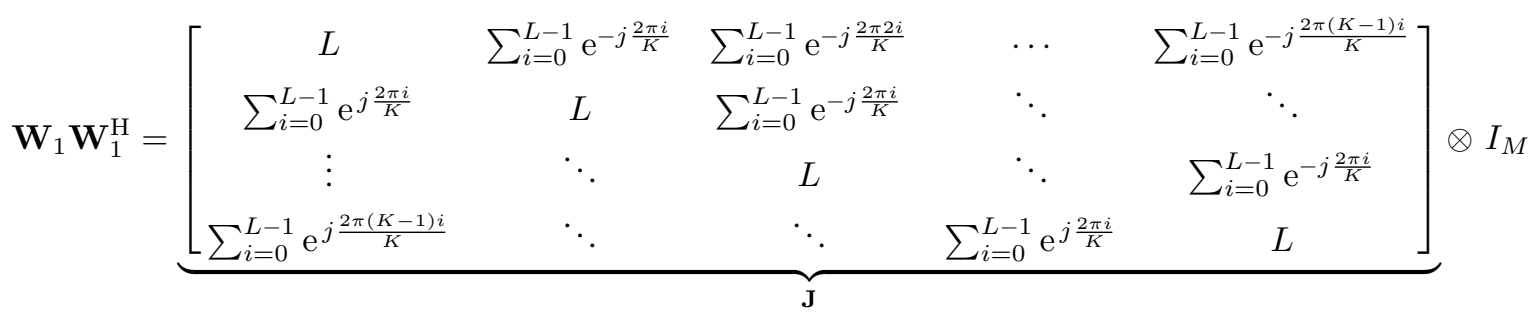


$L=m K, m$ is a positive integer. Hence, $\operatorname{det}\left(\mathbf{W}_{1} \mathbf{W}_{1}^{\mathrm{H}}\right)=$ $\left\{\prod_{n=0}^{K-1}[(\lfloor(L-1-n) / K\rfloor+1) K]\right\}^{M} \leq L^{M K}$. Because

$$
\begin{aligned}
& A_{(i-1) G+g}^{*} A_{(i-1) G+g}^{\mathrm{T}} \\
& =\left[\begin{array}{lll}
\left|\theta_{(i-1) M+1}^{\mathrm{T}}\left(\mathbf{s}_{g}-\tilde{\mathbf{s}}_{g}\right)\right|^{2} & & \\
& \ddots & \\
& & \left|\theta_{i M}^{\mathrm{T}}\left(\mathbf{s}_{g}-\tilde{\mathbf{s}}_{g}\right)\right|^{2}
\end{array}\right]
\end{aligned}
$$

it is easy to prove that

$$
\begin{aligned}
\operatorname{det} & \left(\mathbf{B}_{g}^{\mathrm{H}} \mathbf{B}_{g}\right) \\
= & \operatorname{det}\left(\left[\begin{array}{lll}
A_{g}^{*} A_{g}^{\mathrm{T}} & & \\
& \ddots & \\
& & A_{(K-1) G+g}^{*} A_{(K-1) G+g}^{\mathrm{T}}
\end{array}\right]\right) \\
= & \prod_{k=1}^{M K}\left|\theta_{k}^{\mathrm{T}}\left(\mathbf{s}_{g}-\tilde{\mathbf{s}}_{g}\right)\right|^{2} .
\end{aligned}
$$

Since $\eta_{l c p}=\min _{\left\{\mathbf{s}_{g}, \tilde{\mathbf{s}}_{g}\right\}} \prod_{k=1}^{M K}\left|\theta_{k}^{\mathrm{T}}\left(\mathbf{s}_{g}-\tilde{\mathbf{s}}_{g}\right)\right|^{2}=\left(\Delta_{\min }^{2} / \beta^{2}\right)^{M K}$ [23], we conclude that $\min _{\forall C_{g} \neq E_{g}} \operatorname{det}\left(G_{g} G_{g}^{\mathrm{H}}\right)=\left(\Delta_{\text {min }}^{2} /\right.$ $\left.\beta^{2}\right)^{M K}\left\{\prod_{n=0}^{K-1}[(\lfloor(L-1-n) / K\rfloor+1) K]\right\}^{M} \leq\left[\left(\Delta_{\min }^{2} / \beta^{2}\right)\right.$ $L]^{M K}$.

\section{B. $K>L$}

For $K>L, \operatorname{rank}\left(S_{g}\right)=M L$. Note that $G_{g}\left(C_{g}, E_{g}\right)^{\mathrm{H}}$ $G_{g}\left(C_{g}, E_{g}\right)$ is now a full-rank matrix with size $M L \times M L$, and since $\prod_{i=0}^{\operatorname{rank}\left(S_{g}\right)-1} \lambda_{i}\left(S_{g}\right)=\operatorname{det}\left[G_{g}\left(C_{g}, E_{g}\right)^{\mathrm{H}} G_{g}\left(C_{g}, E_{g}\right)\right]$, the coding gain

$$
G_{g, c}=\min _{\forall C_{g} \neq E_{g}}\left\{\operatorname{det}\left[G_{g}\left(C_{g}, E_{g}\right)^{\mathrm{H}} G_{g}\left(C_{g}, E_{g}\right)\right]\right\}^{\frac{1}{M L}}
$$

where $G_{g}\left(C_{g}, E_{g}\right)^{\mathrm{T}}$ is of size $M L \times M K$ and given by

$$
G_{g}\left(C_{g}, E_{g}\right)^{\mathrm{T}}=\left[\begin{array}{c}
\left(C_{g}-E_{g}\right) \\
{\left[\left(C_{g}-E_{g}\right) D_{\mathrm{sub}, g}\right]} \\
\vdots \\
{\left[\left(C_{g}-E_{g}\right) D_{\mathrm{sub}, g}^{L-1}\right.}
\end{array}\right] .
$$

From the definition of $G_{g}\left(C_{g}, E_{g}\right)^{\mathrm{T}}$, we get (42) shown at the bottom of the page. After some manipulation, the $j$ th column in the last step of (42) can be rewritten as shown in (43) at the bottom of the next page.

Processing each column in the last step of (42) in this way, we get $G_{g}\left(C_{g}, E_{g}\right)^{\mathrm{H}} G_{g}\left(C_{g}, E_{g}\right)$ and is written as $G_{g}^{\mathrm{H}} G_{g}$ for simplicity in the sequel) shown in (44) at the bottom of the next page.

Note: Because the matrix $\mathbf{W}$ (size $M L \times M K$ ) is not square for $K>L$, we have to resort to Ostrowski's Theorem [25], [31] that is quoted here for completeness.

Theorem (Ostrowski) 1: Let $\mathbf{A}$ and $\mathbf{S}$ be $n \times n$ matrices with $\mathbf{A}$ Hermitian and $\mathbf{S}$ nonsingular. Let the eigenvalues of $\mathbf{A}$ and SS $^{\mathrm{H}}$ be arranged in increasing order $\lambda_{\min }=\lambda_{1} \leq \lambda_{2} \leq \cdots \leq$ $\lambda_{n-1} \leq \lambda_{n}=\lambda_{\max }$. For each $k=1,2, \ldots, n$, there exists a nonnegative real number $\theta_{k}$ such that $0<\lambda_{1}\left(\mathbf{S S}^{\mathrm{H}}\right) \leq \theta_{k} \leq$ $\lambda_{n}\left(\mathbf{S S}^{\mathrm{H}}\right)$ and $\lambda_{k}\left(\mathbf{S} \mathbf{A} \mathbf{S}^{\mathrm{H}}\right)=\theta_{k} \lambda_{k}(\mathbf{A})$.

Repeatedly using the properties: 1$) \operatorname{det}(A)=\prod \lambda_{i}(A)$ and 2) for $A(m \times n), B(n \times m), n \geq m, \lambda_{1}, \ldots, \lambda_{m}$ are the eigenvalues of $A B, \longrightarrow \lambda_{1}, \ldots, \lambda_{m}, 0, \ldots, 0$ are the eigenvalues of $B A$ [21], [25] and Ostrowski's Theorem [25], [31], we can get

$\operatorname{det}\left(G_{g}^{\mathrm{H}} G_{g}\right)$

$$
\begin{aligned}
& =\operatorname{det}\left(\mathbf{W} \boldsymbol{\Lambda}_{g} \mathbf{W}^{\mathrm{H}}\right)=\operatorname{det}\left(\mathbf{W} \boldsymbol{\Lambda}_{g}^{\frac{1}{2}} \boldsymbol{\Lambda}_{g}^{\frac{H}{2}} \mathbf{W}^{\mathrm{H}}\right) \\
& =\prod_{i=1}^{M L} \lambda_{i}\left[\mathbf{W} \boldsymbol{\Lambda}_{g}^{\frac{1}{2}}\left(\mathbf{W} \boldsymbol{\Lambda}_{g}^{\frac{1}{2}}\right)^{\mathrm{H}}\right]=\prod_{j=1}^{M L} \lambda_{j}\left(\boldsymbol{\Lambda}_{g}^{\frac{H}{2}} \mathbf{W}^{\mathrm{H}} \mathbf{W} \boldsymbol{\Lambda}_{g}^{\frac{1}{2}}\right) \\
& =\prod_{j=1}^{M L} \beta_{j} \lambda_{j}\left(\mathbf{W}^{\mathrm{H}} \mathbf{W}\right)=\prod_{j=1}^{M L} \beta_{j} \times \prod_{j=1}^{M L} \lambda_{j}\left(\mathbf{W}^{\mathrm{H}} \mathbf{W}\right) \\
& =\prod_{j=1}^{M L} \beta_{j} \times \prod_{j=1}^{M L} \lambda_{j}\left(\mathbf{W} \mathbf{W}^{\mathrm{H}}\right)=\prod_{j=1}^{M L} \beta_{j} \times \operatorname{det}\left(\mathbf{W} \mathbf{W}^{\mathrm{H}}\right),
\end{aligned}
$$

where $0<\lambda_{\min }\left(\boldsymbol{\Lambda}_{g}\right) \leq \beta_{j} \leq \lambda_{\max }\left(\boldsymbol{\Lambda}_{g}\right)$.

Using (38), we can simplify the above to yield $0<$ $\min _{k \in 1, \ldots, M K}\left|\theta_{k}^{\mathrm{T}}\left(\mathbf{s}_{g}-\tilde{\mathbf{s}}_{g}\right)\right|^{2} \leq \beta_{j} \leq \max _{k \in 1, \ldots, M K} \mid \theta_{k}^{\mathrm{T}}\left(\mathbf{s}_{g}-\right.$ $\left.\tilde{\mathbf{s}}_{g}\right)\left.\right|^{2}$.

Next, we evaluate $\operatorname{det}\left(\mathbf{W} \mathbf{W}^{\mathrm{H}}\right)$ in detail in (45) at the bottom of the next page. In (46), we use the property of the matrices

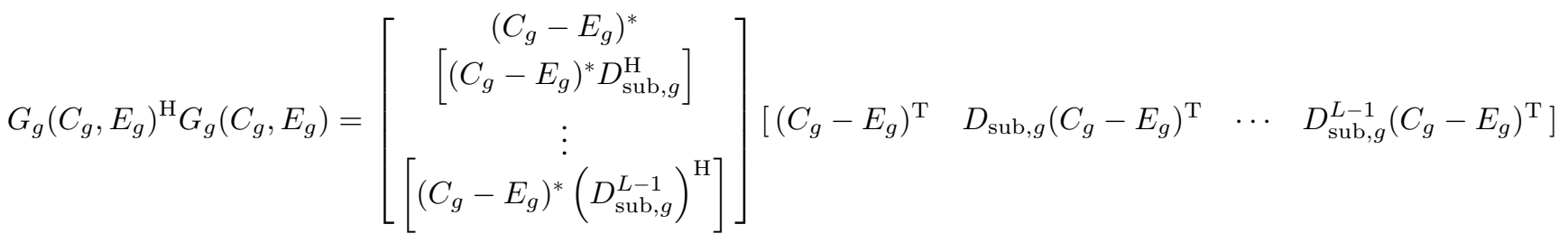

$$
\begin{aligned}
& =\left[\begin{array}{ccc}
\left(C_{g}-E_{g}\right)^{*}\left(C_{g}-E_{g}\right)^{\mathrm{T}} & \cdots & \left(C_{g}-E_{g}\right)^{*} D_{\mathrm{sub}, g}^{L-1}\left(C_{g}-E_{g}\right)^{\mathrm{T}} \\
\left(C_{g}-E_{g}\right)^{*} D_{\mathrm{sub}, g}^{\mathrm{H}}\left(C_{g}-E_{g}\right)^{\mathrm{T}} & \cdots & \left(C_{g}-E_{g}\right)^{*} D_{\mathrm{sub}, g}^{L-2}\left(C_{g}-E_{g}\right)^{\mathrm{T}} \\
\vdots & \ddots & \vdots \\
\left(C_{g}-E_{g}\right)^{*}\left(D_{\mathrm{sub}, g}^{\mathrm{H}}\right)^{L-1}\left(C_{g}-E_{g}\right)^{\mathrm{T}} & \cdots & \left(C_{g}-E_{g}\right)^{*}\left(C_{g}-E_{g}\right)^{\mathrm{T}}
\end{array}\right]
\end{aligned}
$$




$$
\begin{aligned}
& {\left[\begin{array}{cccc}
D_{g}^{j-1} & D_{G+g}^{j-1} & \cdots & D_{(K-1) G+g}^{j-1} \\
D_{g}^{j-2} & D_{G+g}^{j-2} & \cdots & D_{(K-1) G+g}^{j-2} \\
\vdots & \vdots & \vdots & \vdots \\
\left(D_{g}^{\mathrm{H}}\right)^{L-j} & \left(D_{G+g}^{\mathrm{H}}\right)^{L-j} & \cdots & \left(D_{(K-1) G+g}^{\mathrm{H}}\right)^{L-j}
\end{array}\right] \underbrace{\left[\begin{array}{c}
A_{g}^{*} A_{g}^{\mathrm{T}} \\
A_{G+g}^{*} A_{G+g}^{\mathrm{T}} \\
\vdots \\
A_{(K-1) G+g}^{*} A_{(K-1) G+g}^{\mathrm{T}}
\end{array}\right]}_{\mathbf{z}_{g}}} \\
& =\left[\begin{array}{cccc}
I_{M} & I_{M} & \cdots & I_{M} \\
D_{g}^{\mathrm{H}} & D_{G+g}^{\mathrm{H}} & \cdots & D_{(K-1) G+g}^{\mathrm{H}} \\
\vdots & \vdots & \vdots & \vdots \\
\left(D_{g}^{\mathrm{H}}\right)^{L-1} & \left(D_{G+g}^{\mathrm{H}}\right)^{L-1} & \cdots & \left(D_{(K-1) G+g}^{\mathrm{H}}\right)^{L-1}
\end{array}\right]\left[\begin{array}{cccc}
D_{g}^{j-1} & & & \\
& D_{G+g}^{j-1} & & \\
& & \ddots & \\
& & & D_{(K-1) G+g}^{j-1}
\end{array}\right] \mathbf{z}_{g}
\end{aligned}
$$

$G_{g}^{\mathrm{H}} G_{g}=\underbrace{\left[\begin{array}{cccc}I_{M} & I_{M} & \cdots & I_{M} \\ D_{g}^{\mathrm{H}} & D_{G+g}^{\mathrm{H}} & \cdots & D_{(K-1) G+g}^{\mathrm{H}} \\ \vdots & \vdots & \vdots & \vdots \\ \left(D_{g}^{\mathrm{H}}\right)^{L-1} & \left(D_{G+g}^{\mathrm{H}}\right)^{L-1} & \cdots & \left(D_{(K-1) G+g}^{\mathrm{H}}\right)^{L-1}\end{array}\right]}_{\mathbf{W}}$

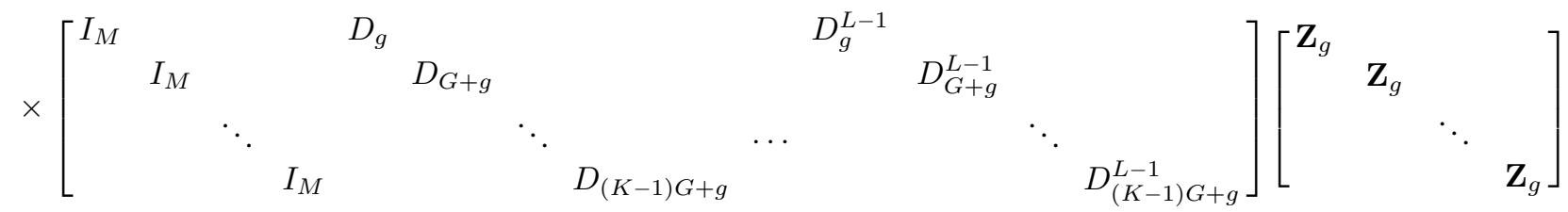

$$
\begin{aligned}
& =\mathbf{W}\left[\begin{array}{ccc}
A_{g}^{*} A_{g}^{\mathrm{T}} & & \\
& \ddots & \\
& & A_{(K-1) G+g}^{*} A_{(K-1) G+g}^{\mathrm{T}}
\end{array}\right]\left[\begin{array}{cccc}
I_{M} & D_{g} & \cdots & D_{g}^{L-1} \\
I_{M} & D_{G+g} & \cdots & D_{G+g}^{L-1} \\
\vdots & \vdots & \vdots & \vdots \\
I_{M} & D_{(K-1) G+g} & \cdots & D_{(K-1) G+g}^{L-1}
\end{array}\right] \\
& =\mathbf{W} \underbrace{\left[\begin{array}{lll}
A_{g}^{*} A_{g}^{\mathrm{T}} & & \\
& \ddots & \\
& & A_{(K-1) G+g}^{*} A_{(K-1) G+g}^{\mathrm{T}}
\end{array}\right]}_{\boldsymbol{\Lambda}_{g}} \mathbf{W}^{\mathrm{H}}
\end{aligned}
$$

$$
\begin{aligned}
\mathbf{W} \mathbf{W}^{\mathrm{H}}= & {\left[\begin{array}{cccc}
I_{M} & I_{M} & \cdots & I_{M} \\
D_{g}^{\mathrm{H}} & D_{G+g}^{\mathrm{H}} & \cdots & D_{(K-1) G+g}^{\mathrm{H}} \\
\vdots & \vdots & \vdots & \vdots \\
\left(D_{g}^{\mathrm{H}}\right)^{L-1} & \left(D_{G+g}^{\mathrm{H}}\right)^{L-1} & \cdots & \left(D_{(K-1) G+g}^{\mathrm{H}}\right)^{L-1}
\end{array}\right]\left[\begin{array}{cccc}
I_{M} & D_{g} & \cdots & D_{g}^{L-1} \\
I_{M} & D_{G+g} & \cdots & D_{G+g}^{L-1} \\
\vdots & \vdots & \vdots & \vdots \\
I_{M} & D_{(K-1) G+g} & \cdots & D_{(K-1) G+g}^{L-1}
\end{array}\right] } \\
= & {\left[\begin{array}{cccc}
K I_{M} & \sum_{i=0}^{K-1} D_{i G+g} & \cdots & \sum_{i=0}^{K-1} D_{i G+g}^{L-1} \\
\sum_{i=0}^{K-1} D_{i G+g}^{-1} & K I_{M} & & \sum_{i=0}^{K-1} D_{i G+g}^{L-2} \\
\vdots & & \ddots & \\
\sum_{i=0}^{K-1} D_{i G+g}^{-(L-1)} & & \cdots & K I_{M}
\end{array}\right] } \\
= &
\end{aligned}
$$


$\left\{D_{i}\right\}$ 's that $D_{i}^{\mathrm{H}}=D_{i}^{-1}$. Equation (47) is the result of $D_{i G+g}=\exp \left[j\left(2 \pi / N_{\mathrm{c}}\right) M G i\right] D_{g}=\exp [j(2 \pi / K) i] D_{g} \quad$ and $\sum_{i=0}^{K-1} \exp [j(2 \pi i l / K)]=0$, where $0<l \leq L-1 \leq K-1$.

Therefore

$$
\operatorname{det}\left(\mathbf{W} \mathbf{W}^{\mathrm{H}}\right)=K^{M L}
$$

Inserting (48) into (40), and using $\operatorname{det}\left(G_{g}^{\mathrm{H}} G_{g}\right)=$ $\operatorname{det}\left(\mathbf{W} \mathbf{W}^{\mathrm{H}}\right) \times \prod_{j=1}^{M L} \beta_{j}$, we get

$$
G_{g, c}=\min _{\forall C_{g} \neq E_{g}}\left[K \times\left(\prod_{j=1}^{M L} \beta_{j}\right)^{\frac{1}{M L}}\right] .
$$

\section{C. $K=L$}

In this case, starting from (40), and noting that in (44), the matrix $\mathbf{W}$ is now square and allows the use of the property that $A, B(m \times m)$, $\operatorname{det}(A B)=\operatorname{det}(A) \operatorname{det}(B)=\operatorname{det}(B A)$; hence, $\operatorname{det}\left(G_{g}^{\mathrm{H}} G_{g}\right)$ can be exactly evaluated, thereby obviating the need for Ostrowski's Theorem. Thus $\operatorname{det}\left(G_{g}^{\mathrm{H}} G_{g}\right)=$ $\operatorname{det}\left(\mathbf{W} \boldsymbol{\Lambda}_{g} \mathbf{W}^{\mathrm{H}}\right)=\operatorname{det}\left(\mathbf{W} \mathbf{W}^{\mathrm{H}}\right) \operatorname{det}\left(\boldsymbol{\Lambda}_{g}\right)=L^{M L} \operatorname{det}\left(\boldsymbol{\Lambda}_{g}\right)$ where (48) with $K=L$ was used in the final step. Using (39), the definition of $\eta_{l c p}$, and noting that for the case $K=L$, $\eta_{l c p}=\left[\Delta_{\min }^{2} / \beta^{2}\right]^{M L}[23]$, we finally get

$$
G_{g, c}=L \times \frac{\Delta_{\min }^{2}}{\beta^{2}} .
$$

\section{REFERENCES}

[1] IEEE Standard for Local and Metropolitan Area Networks-Pt. 16: Air Interface for Fixed Broadband Wireless Access Systems, IEEE 802.162001, Apr. 2002.

[2] Draft Amendment to IEEE Standard for Local and Metropolitan Area Networks-Pt. 16: Air Interface for Fixed Broadband Wireless Access Systems- Medium Access Control Modifications and Additional Physical Layer Specifications for 2-11 GHz, IEEE P802.16a/D3-2001, Mar. 2002.

[3] H. Bolcskei and A. J. Paulraj, "Space-frequency coded broadband OFDM systems," in Proc. IEEE Wireless Communications and Networking Conf. (WCNC), Chicago, IL, 2000, vol. 1, pp. 1-6.

[4] _ "Space-frequency codes for broadband fading channels," in Proc. IEEE Int. Symp. Information Theory (ISIT), Washington, DC, 2001, p. 219.

[5] Z. Liu, Y. Xin, and G. B. Giannakis, "Space-time-frequency coded OFDM with sub-carrier grouping and constellation precoding," in Proc. Int. Conf. Acoustics, Speech and Signal Processing (ASSP), Orlando, FL, 2002, vol. 3, pp. 2205-2208.

[6] B. Lu and X. Wang, "Space-time code design in OFDM systems," in IEEE Global Telecommunications Conf. (GLOBECOM), San Francisco, CA, 2000, vol. 2, pp. 1000-1004.

[7] Y. Xin, Z. Wang, and G. B. Giannakis, "Linear unitary precoders for maximum diversity gains with multiple transmit- and receive-antennas," in Proc. 34th Asilomar Conf. Signals, Systems, and Computers (Invited Paper), Pacific Grove, CA, 2000, pp. 1553-1557.

[8] _ , "Space-time constellation-rotating codes maximizing diversity and coding gains," in Proc. Global Telecommunications (GLOBECOM), San Antonio, TX, 2001, vol. 1, pp. 455-459.

[9] L. Zheng and D. N. C. Tse, "Diversity and multiplexing: A fundamental tradeoff in multiple-antenna channels," IEEE Trans. Inf. Theory, vol. 49, no. 5, pp. 1073-1096, May 2003.
[10] D. Agrawal, V. Tarokh, A. Naguib, and N. Seshadri, "Space-time coded OFDM for high data-rate wireless communication over wideband channels," in Proc. Vehicular Technology Conf. (VTC), Ottawa, ON, Canada, 1998, pp. 2232-2236.

[11] M. O. Damen, A. Chkeif, and J.-C. Belfiore, "Lattice codes decoder for space-time codes," IEEE Commun. Lett., vol. 4, no. 5, pp. 161-163, May 2000.

[12] K. F. Lee and D. B. Williams, "A space-frequency transmitter diversity technique for OFDM systems," in Proc. IEEE Global Telecommunications Conf. (GLOBECOM), San Francisco, CA, 2000, vol. 3, pp. 1473-1477.

[13] Z. Liu, G. B. Giannakis, B. Muquet, and S. Zhou, "Space-time coding for broadband wireless communications," in Wireless Commun. Mob. Comput., vol. 1, no. 1, New York: Wiley, Jan.-Mar. 2001, pp. 33-53.

[14] P. Joo, J. Son, and D. Kang, "Analysis of STFBC-OFDM for BWA in SUI Channel," Samsung Electronics, Korea, IEEE C802.16a-02/18, Jan. 22, 2002.

[15] S. M. Alamouti, "A simple transmit diversity technique for wireless communications," IEEE J. Sel. Areas Commun., vol. 16, no. 8, pp. 1451-1458, Oct. 1998.

[16] R. J. Piechocki, P. N. Fletcher, A. R. Nix, C. N. Canagarajah, and J. P. McGeehan, "Performance evaluation of BLAST-OFDM enhanced Hiperlan/2 using simulated and measured channel data," Electron. Lett., vol. 37, no. 18, pp. 1137-1139, Aug. 30, 2001.

[17] A. M. Chan and I. Lee, "A new reduced-complexity sphere decoder for multiple antenna systems," in IEEE Int. Conf. Communications (ICC), New York, 2002, vol. 1, pp. 460-464.

[18] V. Tarokh, H. Jafarkhani, and A. R. Calderbank, "Space-time block codes from orthogonal designs," IEEE Trans. Inf. Theory, vol. 45, no. 5, pp. 1456-1467, Jul. 1999.

[19] D. Gore, S. Sandhu, and A. J. Paulraj, "Delay diversity codes for frequency selective channels," in IEEE Int. Conf. Communications (ICC), New York, 2002, vol. 3, pp. 1949-1953.

[20] X. Ma and G. B. Giannakis, "Space-time-multipath coding using digital phase sweeping," in Proc. Global Telecommunications Conf. (GLOBECOM), Taipei, Taiwan, R.O.C., 2002, pp. 384-388.

[21] H. Lutkepohl, Handbook of Matrices. New York: Wiley, 1996.

[22] Y. Xin, Z. Wang, and G. B. Giannakis, "Space-time diversity systems based on linear constellation precoding," IEEE Trans. Wireless Commun., vol. 2, no. 2, pp. 294-309, Mar. 2003.

[23] Z. Liu, Y. Xin, and G. B. Giannakis, "Linear constellation precoding for OFDM with maximum multipath diversity and coding gains," IEEE Trans. Commun., vol. 51, no. 3, pp. 416-427, Mar. 2003.

[24] _ "Space-time-frequency coded OFDM over frequency-selective fading channels," IEEE Trans. Signal Process., vol. 50, no. 10, pp. 24652476, Oct. 2002.

[25] H. Bolcskei, M. Borgmann, and A. J. Paulraj, "Impact of the propagation environment on the performance of space-frequency coded MIMO-OFDM," IEEE J. Sel. Areas Commun., vol. 21, no. 3, pp. 427439, Apr. 2003.

[26] M. O. Damen and N. C. Beaulieu, "A study of some space-time codes with rates beyond one symbol per channel use," in IEEE Global Telecommunications Conf. (GLOBECOM), San Antonio, TX, 2001, vol. 1, pp. $445-449$.

[27] O. Tirkkonen and A. Hottinen, "Improved MIMO performance with nonorthogonal space-time block codes," in IEEE Global Telecommunications Conf. (GLOBECOM), San Antonio, TX, 2001, pp. 1122-1126.

[28] H. El Gamal and M. O. Damen, "An algebraic number theoretic framework for space-time coding," in Proc. IEEE Int. Symp. Information Theory (ISIT), Lausanne, Switzerland, 2002, p. 132.

[29] O. Tirkkonen, A. Boariu, and A. Hottinen, "Minimal non-orthogonality rate 1 space-time block code for 3+ Tx antennas," in IEEE 6th Int. Symp. Spread Spectrum Techniques and Applications, Parsippany, NJ, 2000, vol. 2, pp. 429-432.

[30] H. Jafarkhani, "A quasi-orthogonal space-time block code," IEEE Trans. Commun., vol. 49, no. 1, pp. 1-4, Jan. 2001.

[31] R. A. Horn and C. R. Johnson, Matrix Analysis. Cambridge, U.K.: Cambridge Univ. Press, 1985.

[32] H. Bolcskei, M. Borgmann, and A. J. Paulraj, "Space-frequency coded MIMO-OFDM with variable multiplexing-diversity tradeoff," in IEEE Int. Conf. Communications (ICC), Anchorage, AK, 2003, vol. 4, pp. 2837-2841. 


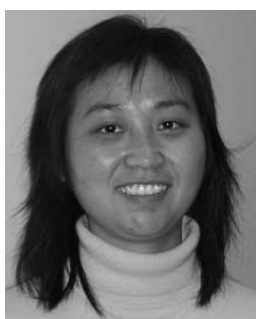

Lei Shao (S'01-M'05) was born in Nanjing, China. She received the B.E. degree from Nanjing University of Posts and Telecommunications in 1997 and the M.E. degree from Southeast University in 2000, both in electrical engineering. In 2004, she received the $\mathrm{Ph} . \mathrm{D}$. degree in electrical engineering at the University of Washington, Seattle.

She was with the Department of Electrical Engineering at the University of Washington. She is with Intel Corporation in Santa Clara, CA. Her research interests are in wireless communications and digital signal processing, including space-time coding and processing for broadband wireless communications, channel estimation for MIMO-OFDM.

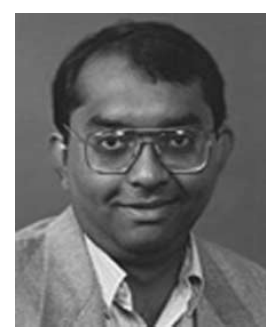

Sumit Roy (S'84-M'88-SM'00) received the B.Tech. degree from the Indian Institute of Technology, Kanpur, India, in 1983, and the M.S. and Ph.D. degrees from the University of California, Santa Barbara, all in electrical engineering in 1985 and 1988 , respectively, as well as an M.A. in statistics and applied probability in 1988.

His previous academic appointments were at the Moore School of Electrical Engineering, University of Pennsylvania, and at the University of Texas, San Antonio. He spent 2001-2003 on academic leave at Intel Wireless Technology Lab working on research and standards development for ultra-wideband radio technology (wireless PANs) and next generation highspeed wireless LANs. Presently, he is Professor of Electrical Engineering, University of Washington, Seattle, where his research interests include analysis/design of communication systems/networks, with a topical emphasis on next generation mobile/wireless networks.

His activities for the IEEE Communications Society includes membership of several technical and conference program committees; he recently completed a term as Editor for the IEEE TRANSACTIONS ON WIRELESS Communications and currently serves on the Editorial Board for Wiley $J$. Wireless Communications and Mobile Computing. 